\title{
Many-body localization in waveguide quantum electrodynamics
}

\author{
N. Fayard $\odot,{ }^{1, *}$ L. Henriet, ${ }^{2}$ A. Asenjo-Garcia, ${ }^{3}$ and D. E. Chang ${ }^{1,4}$ \\ ${ }^{1}$ ICFO-Institut de Ciències Fotòniques, The Barcelona Institute of Science and Technology, 08860 Castelldefels, Barcelona, Spain \\ ${ }^{2}$ Pasqal, 2 av. Augustin Fresnel, 91120 Palaiseau, France \\ ${ }^{3}$ Department of Physics, Columbia University, New York, New York 10027, USA \\ ${ }^{4}$ ICREA-Institució Catalana de Recerca i Estudis Avançats, 08015 Barcelona, Spain
}

(Received 3 April 2021; revised 9 August 2021; accepted 12 August 2021; published 10 September 2021)

\begin{abstract}
At the quantum many-body level, atom-light interfaces generally remain challenging to solve for or understand in a nonperturbative fashion. Here we consider a waveguide quantum electrodynamics model, where two-level atoms interact with and via propagating photons in a one-dimensional waveguide, and specifically investigate the interplay of atomic position disorder, multiple scattering of light, quantum nonlinear interactions, and dissipation. We develop qualitative arguments and present numerical evidence that such a system exhibits a many-body localized (MBL) phase, provided that atoms are less than half excited. Interestingly, while MBL is originally formulated with respect to closed systems, this system is intrinsically open. However, as dissipation originates from transport of energy to the system boundaries and the subsequent radiative loss, the lack of transport in the MBL phase makes the waveguide QED system look essentially closed and makes applicable the notions of MBL. Conversely, we show that if the system is initially in a delocalized phase due to a large excitation density, then rapid initial dissipation can leave the system unable to efficiently transport energy at later times, resulting in a dynamical transition to an MBL phase. These phenomena can be feasibly realized in state-of-the-art experimental setups.
\end{abstract}

DOI: 10.1103/PhysRevResearch.3.033233

\section{INTRODUCTION}

Quantum light-matter interfaces are being actively investigated, for their many possibilities to explore fundamental science and for applications [1,2]. However, the full quantum many-body problem of interacting light and matter remains highly challenging to understand, not only due to the large Hilbert space but also its out-of-equilibrium and open nature. The interplay between different effects, possibly including strength of interactions, correlated emission, dimensionality, and/or randomness, can give rise to a wealth of different relaxation dynamics for initially excited systems. This ranges from the collective emission effects of superradiance [3] and subradiance $[4,5]$ in atomic ensembles to critical slow-down in atomic arrays [6] to the formation of exciton polariton condensates in microcavities [7] to the stabilization of photonic Mott insulators [8].

Within condensed matter physics, an exciting new paradigm for relaxation, or more specifically the lack thereof, has emerged in recent years. In particular, many-body localization (MBL) is a phenomenon in which a closed, interacting system can fail to thermalize [9-13]. Instead, the system exhibits a number of distinct properties, including the absence of energy transport given an arbitrary initial excited state

\footnotetext{
*Corresponding author: fayardnikos@gmail.com

Published by the American Physical Society under the terms of the Creative Commons Attribution 4.0 International license. Further distribution of this work must maintain attribution to the author(s) and the published article's title, journal citation, and DOI.
}

and thus a strong memory of initial conditions, and the slow growth of entanglement entropy following a quench. Lying outside of the scope of equilibrium descriptions of matter, MBL can be considered as a novel dynamical phase.

Here we propose the existence and theoretically investigate the properties of MBL in a system involving atoms and light. Specifically, we consider a minimal "waveguide quantum electrodynamics (QED)" model consisting of disordered two-level atoms or qubits interacting with photons in an infinite one-dimensional (1D) waveguide. We provide qualitative arguments and quantitative numerical simulations to argue that the system can support a many-body localized phase. While the concept of MBL is originally formulated with respect to closed systems, our waveguide QED system is intrinsically open, as light emitted by any finite set of excited atoms will be irreversibly lost once passing the boundaries of the atomic ensemble. Generically, one would expect that such spontaneous emission will result in the atoms to eventually return to their ground states (although the dynamics itself can be made richer by the collective nature of emission [3-6]). However, we find that this open nature does not simply destroy MBL, but instead results in an additional rich interplay between dissipation and essentially closed, MBL-like dynamics [14-18]. In particular, we show that an MBL phase occurs at low atomic excitation densities (approximately less than half excited in the large atom limit). The absence of transport then suppresses propagation of energy to the system boundaries and the resulting dissipation, which in turn makes the notion of MBL applicable in the first place. On the other hand, for systems that are initially highly excited, transport can be responsible for a rapid dissipation at early times. This causes the atomic excitation density to drop until transport is no longer 
(a)

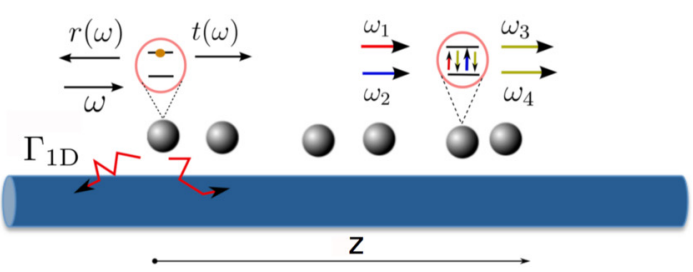

(c)

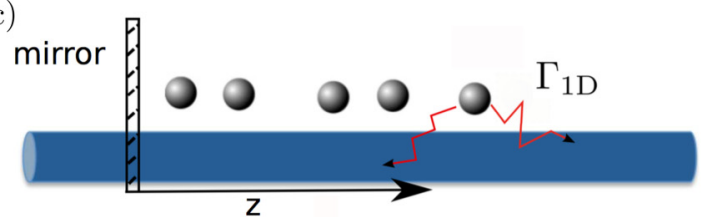

(b)

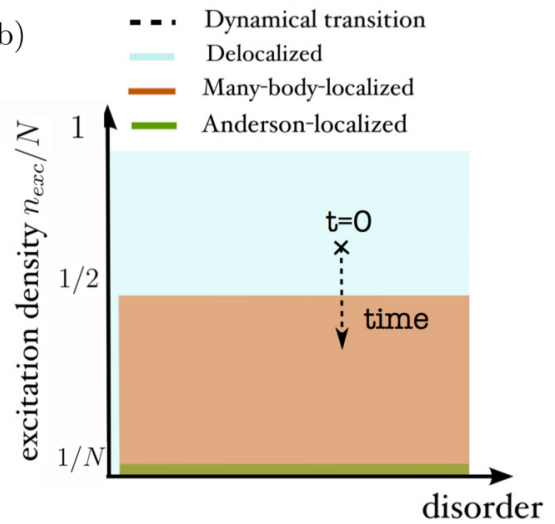

FIG. 1. (a) Schematic illustration of a spatially disordered atomic chain coupled to photons in a one-dimensional, bidirectional waveguide. The magnitude of atom-light interactions is set by the rate $\Gamma_{1 \mathrm{D}}$ by which a single, isolated excited atom irreversibly emits a photon into the waveguide. With multiple atoms and excitations, the system dynamics becomes complex and interacting due to the following possibilities. First, a photon can be reflected or transmitted with nontrivial, frequency-dependent amplitudes $r(\omega), t(\omega)$ upon scattering off of an atom, which then leads to multiple scattering in the presence of multiple atoms. Second, multiple photons (e.g., two photons with frequencies $\omega_{1,2}$ illustrated) scattering off of the same atom can lead to the generation of entangled, frequency-mixed photons $\left(\omega_{3}+\omega_{4}=\omega_{1}+\omega_{2}\right)$, which also become multiply scattered. (b) Proposed phase diagram in the thermodynamic limit of large atom number $N \rightarrow \infty$, versus amount of disorder and fraction of atoms excited, $n_{\text {exc }} / N$. For a single excitation, $n_{\text {exc }} / N=1 / N$, the system is noninteracting, and arbitrarily small disorder gives rise to Anderson localization. Beyond a single excitation, the system becomes interacting due to the nonlinearity of two-level atoms. Up to $n_{\text {exc }} / N=1 / 2$, we hypothesize that the system exhibits MBL. For $n_{\text {exc }} / N>1 / 2$, photon transport is allowed and leads to delocalization. Going beyond the thermodynamic limit, and considering a finite atomic system, transport in the delocalized phase leads to dissipation, in the form of emission of light beyond the atomic system boundaries. The subsequent loss of excitation density can lead to a dynamical transition into the MBL phase, as depicted by the dashed arrow showing time evolution. (c) Schematic illustration of a spatially disordered atomic chain coupled to photons in a one-dimensional, "half" waveguide, with one end closed by a mirror at $z=0$. The atomic system can thus only dissipate by emitting photons from one side of the chain.

efficient, resulting in a dynamical transition into an $\mathrm{MBL}$ phase. This physics should be feasible to explore in existing state-of-the-art waveguide QED systems, such as superconducting quantum bits coupled to microwave transmission lines [19-21] or structured waveguides [22,23].

An additional notable feature of our work, within the context of atom-light interactions, is that the MBL phase arises from the combination of atomic granularity, disorder, strong nonlinearities, and multiple scattering of light. There has been significant work aimed at understanding the consequences of granularity and multiple scattering in recent years [5,2436] but largely restricted to classical or mean-field limits, where potentially strong nonlinear optical interactions and the buildup of quantum correlations are ignored. MBL thus constitutes an interesting limit where the physics emerging from strong interactions can be well understood in a nonperturbative fashion.

The rest of the paper is organized as follows. First, in Sec. II, we introduce the waveguide QED Hamiltonian and present a qualitative argument that an MBL phase should exist in the thermodynamic limit, provided that the atoms are less than half excited. Given the large Hilbert space of the system, consisting of the exponentially large space associated with the atoms and the infinite space associated with the continuum of photon modes, we then restrict ourselves to the regime of near-resonant photon interactions. Then, in Sec. III, we show how the photonic modes can be integrated out, reducing the system to an open, interacting "spin model" describing photon-mediated interactions between the atomic degrees of freedom. Before going to MBL, and to gain intuition, we show how Anderson localization manifests itself in the spin model formalism, within the linear optics (single-excitation) limit. This example illustrates that although the system is formally open, localization implies that dynamics of a large system is well described just by the Hermitian Hamiltonian component of the spin model. We briefly introduce the phenomenology of MBL phases in Sec. IV. As MBL is typically studied with respect to closed systems, and given the observation above regarding the connection between localization and Hermitianity, in Sec. V we present numerical evidence of MBL for the Hermitian Hamiltonian, namely the absence of transport and logarithmic growth of entanglement entropy for sufficiently low excitation densities. In Sec. VI we then consider the physical open system and propose and numerically investigate a number of realistic observables for MBL. At low excitation densities, we again find an MBL phase. Surprisingly, however, for higher excitation densities, where MBL is not observed for the closed system, we find that transport to the system boundaries and the corresponding dissipation enable the system to initially and rapidly lower the excitation density, and thus dynamically transition into an MBL phase. Finally, in Sec. VII, we provide an outlook of possible future interesting directions to explore and discuss the prospects of experimentally observing such physics in state-of-the-art waveguide QED platforms.

\section{DESCRIPTION OF THE SYSTEM}

Our system of interest is represented in Fig. 1(a). It consists of a one-dimensional, bidirectional waveguide, whose photons couple to the ground-excited $(|g\rangle-|e\rangle)$ transitions of 
$N$ two-level atoms located at random positions $z_{i}$. The atomic transition frequency is given by $\omega_{0}$. A minimal model for such a system is given by the following Hamiltonian (with $\hbar=1$ ):

$$
\begin{aligned}
\mathcal{H}= & \underbrace{\omega_{0} \sum_{i} \hat{\sigma}_{e e}^{i}}_{\mathcal{H}_{\mathrm{a}}}+\underbrace{\sum_{\nu= \pm} \int d k \omega_{k} \hat{b}_{v, k}^{\dagger} \hat{b}_{v, k}}_{\mathcal{H}_{\mathrm{ph}}} \\
& +\underbrace{g \sum_{\nu, i} \int d k\left(\hat{b}_{\nu, k} \hat{\sigma}_{e g}^{i} e^{i \nu k z_{i}}+\text { H. c. }\right)}_{\mathcal{H}_{\mathrm{int}}} .
\end{aligned}
$$

Here $\mathcal{H}_{\mathrm{a}}$ describes the excited-state energy of the atoms, and we adopt the notation $\hat{\sigma}_{\alpha \beta}^{i}=\left|\alpha_{i}\right\rangle\left\langle\beta_{i}\right|$ with $\{\alpha, \beta\} \in\{g, e\}$ for the operators of atom $i$. $\mathcal{H}_{\mathrm{ph}}$ describes the energy of the photons, characterized by a continuum of modes with two possible propagation directions (labeled $v= \pm$ ) and wave vector $k$. We assume that within the bandwidth of modes to which the atoms significantly couple, the dispersion relation for the guided modes can be linearized as $\omega_{k}=c|k|$. The interactions, as given by $\mathcal{H}_{\text {int }}$, describe the processes by which an excited atom can emit a photon, or a ground-state atom can absorb a photon, with a coupling constant $g$ assumed to be identical for all atoms, and an interaction phase $e^{i v k z_{i}}$ encoding the photon propagation. In the following, we consider disorder in the positions of the atoms: $z_{i}=\left(i+\epsilon_{i}\right) d$, with $d$ the average distance between two neighboring atoms and $\epsilon_{i}$ a random variable. For numerics, we will focus on the regime of full disorder, where $\epsilon_{i}$ is drawn between $-1 / 2$ and $1 / 2$, in order to minimize the effective localization length of the system and the number of atoms needed in simulations, although we believe our conclusions are general for any amount of disorder (see below). The Hamiltonian of Eq. (1) can be an excellent approximation for a number of systems such as superconducting qubits coupled to a transmission line [19-21], or atoms coupled to a photonic crystal waveguide [37-39], where additional sources of dissipation (not included in this model) can potentially be much smaller than the coherent atom-waveguide interactions.

To gain some basic insight into Eq. (1), we begin by relating it to well-known results regarding Anderson localization of light in $1 \mathrm{D}$, in the linear optical or single-excitation limit with disorder in the position of the atoms. The single-photon, single-atom scattering dynamics of Eq. (1) can be exactly solved [40,41]. In particular, the response of a ground-state atom to an incoming photon of well-defined frequency $\omega$ is characterized by the reflection and transmission coefficients $r(\omega)=-\Gamma_{1 \mathrm{D}} /\left(\Gamma_{1 \mathrm{D}}-2 i \Delta\right)$ and $t(\omega)=1+r(\omega)$ [Fig. 1(a)], with $\Delta=\omega-\omega_{0}$ being the detuning between the photon and the atomic transition, and $\Gamma_{1 \mathrm{D}}=4 \pi g^{2} / c$ being the spontaneous emission rate of a single, isolated excited atom into the waveguide. When many atoms are coupled together via the waveguide, the multiple scattering arising from the succession of transmission and reflection events [Fig. 1(a)] by different atoms can be solved by the transfer matrix formalism [42]. Performing a disorder average over the position fluctuations $\epsilon_{i}$ of the $\mathrm{N}$ atoms, the 1D waveguide is always Anderson localized for arbitrarily small disorder [arbitrarily narrow distributions of $\epsilon_{i}$, see Fig. 1(b)] [43-46]. The transmittance exhibits exponential attenuation with large fluc- tuations $\overline{\log \left(T_{\text {tot }}\right)}=-N / N_{\text {loc }}$ and $\operatorname{var}\left[\log \left(T_{\text {tot }}\right)\right]=2 N / N_{\text {loc }}$, with $\overline{(\ldots)}$ denoting the average value over the different disorder realizations and $\operatorname{var}(\ldots)$ the variance of the random variable. $N_{\text {loc }}$ is the localization length (expressed in terms of number of atoms) and is related to the single-atom linear transmittance $T(\Delta)=|t(\Delta)|^{2}$ by $N_{\text {loc }}(\Delta)=1 /|\log T(\Delta)|=$ $\left|\log \left[4 \Delta^{2} /\left(\Gamma_{1 \mathrm{D}}^{2}+4 \Delta^{2}\right)\right]\right|^{-1}$ in the dilute regime $(d>\lambda=$ $2 \pi c / \omega)$ and for large disorder [42]. This quantity goes to zero at resonance and increases with increasing detuning $|\Delta|$ as the atomic response becomes weaker away from resonance. For systems larger than the localization length $N>N_{\text {loc }}$, strong destructive interference suppresses the propagation of light through the medium.

While rigorously establishing an MBL transition beyond the single-excitation limit is more complicated, we can nonetheless make a qualitative argument, by calculating a nonlinear single-atom transmittance $T\left(\Delta, \rho_{e e}\right)$ that depends on the excited-state population $\rho_{e e}$, and then substituting into the Anderson localization result $N_{\text {loc }}\left(\Delta, \rho_{e e}\right) \sim$ $1 /\left|\log T\left(\Delta, \rho_{e e}\right)\right|$ to estimate a length scale over which transport becomes prohibited. As discussed in the Appendix, this transmittance can be exactly calculated for an incident coherent state field of arbitrary amplitude, with corresponding Rabi frequency $\Omega$. One finds the relations $\rho_{e e}=\frac{\Omega^{2}}{\Gamma_{1 \mathrm{D}}^{2}+4 \Delta^{2}+2 \Omega^{2}}$ and $T=\frac{4 \Delta^{2}+8 \Omega^{2}}{\Gamma_{\text {ID }}^{2}+4 \Delta^{2}+8 \Omega^{2}}$. Notably, for large photon input $(\Omega \rightarrow \infty)$, one finds that $\rho_{e e} \rightarrow 1 / 2$ and $T \rightarrow 1$. This expresses the wellknown result that an atom becomes saturated at very high intensities and is no longer able to respond to light [47]. In turn, reflection and multiple scattering are suppressed, and the localization length $N_{\text {loc }}\left(\Delta, \rho_{e e} \rightarrow 1 / 2\right) \rightarrow \infty$ diverges. We thus hypothesize that an atomic excitation density of $n_{\text {exc }} / N=$ $1 / 2$ sets the MBL-delocalization transition, as illustrated in Fig. 1(b).

\section{SPIN MODEL}

Beyond a single excitation, directly solving Eq. (1) is difficult due to the infinite Hilbert space of the photons and frequency mixing induced by the atoms $[48,49]$. Furthermore, the localization length $N_{\text {loc }}\left(\Delta, \rho_{e e}\right)$ increases both with increasing detuning and atomic population, and can rapidly exceed numerically tractable system sizes (see Appendix).

To partially mitigate these challenges, we will restrict ourselves to the regime in which the photons involved in the dynamics are near resonance with the atoms. As discussed in Ref. [50], since the field part of the Hamiltonian (1) is quadratic, it can be formally integrated out, resulting in photon-mediated interactions between atoms. Furthermore, near resonance, the delay time of these interactions due to the speed of light can be ignored to good approximation. This is because the atoms in typical situations have very large ratios of resonance frequencies to linewidths, $\omega_{0} \gg \Gamma_{1 \mathrm{D}}$. Thus, the atoms are very dispersive, for example, as characterized by the large, frequency-dependent phase shifts in $r(\omega), t(\omega)$, and this causes the propagation delay of near-resonant photons to be dominated by interaction with the atoms, rather than the speed of light itself. Equivalently, the hybrid atom-photon polaritons that diagonalize the system are in fact almost entirely atomic in nature. Ignoring retardation, one obtains a reduced master 
equation describing instantaneous photon-mediated interactions, between only the atomic "spin" degrees of freedom [50-57],

$$
\dot{\hat{\rho}}=-i\left[\mathcal{H}_{1 \mathrm{D}} \hat{\rho}-\hat{\rho} \mathcal{H}_{1 \mathrm{D}}^{\dagger}\right]+\sum_{i, j} \Gamma_{i, j} \hat{\sigma}_{g e}^{i} \hat{\rho} \hat{\sigma}_{e g}^{j},
$$

with

$$
\mathcal{H}_{1 \mathrm{D}}=-i \frac{\Gamma_{1 \mathrm{D}}}{2} \sum_{i, j=1}^{N} \exp \left(-i k_{1 \mathrm{D}}\left|z_{i}-z_{j}\right|\right) \hat{\sigma}_{e g}^{i} \hat{\sigma}_{g e}^{j}
$$

and $\Gamma_{i, j}=\Gamma_{1 \mathrm{D}} \cos \left(k_{1 \mathrm{D}}\left|z_{i}-z_{j}\right|\right)$ and $k_{1 \mathrm{D}}=\omega_{0} / c$. Here we have transformed to a rotating frame, so that the trivial phase evolution associated with $\mathcal{H}_{\mathrm{a}}$ can be ignored. We note that in one dimension, the photon-mediated interactions between atoms are infinite in range. Furthermore, integrating out the photons results in a dissipative (open) system, as atoms can physically emit a photon that goes beyond the atomic system boundaries, resulting in irreversible loss of excited population.

If loss is indeed associated with the system boundaries, then conceptually its effects (for a fixed initial number of excitations) can be reduced by considering progressively larger system sizes, where the ratio of "boundary" (e.g., atoms within a distance $\sim N_{\text {loc }}$ of an edge) to "bulk" regions decreases. While the maximum number of atoms in numerics is constrained, we can nonetheless decrease this ratio by introducing a closely related "half-waveguide" model, as illustrated in Fig. 1(c). Here one end of the waveguide (say, at $z=0$ ) is terminated by a perfect mirror, which results in only one open boundary where photons can escape. Applying similar considerations as the derivation of Eq. (2), it is straightforward to show that the master equation is

$$
\dot{\hat{\rho}}=-i\left[\mathcal{H}_{\text {half }} \hat{\rho}-\hat{\rho} \mathcal{H}_{\text {half }}^{\dagger}\right]+\sum_{i, j} \Gamma_{i, j} \hat{\sigma}_{g e}^{i} \hat{\rho} \hat{\sigma}_{e g}^{j},
$$

with

$$
\begin{aligned}
\mathcal{H}_{\text {half }}= & -i \Gamma_{1 \mathrm{D}} / 2 \sum_{i, j=1}^{N}\left[\exp \left(-i k_{1 \mathrm{D}}\left|z_{i}-z_{j}\right|\right)\right. \\
& \left.-\exp \left(-i k_{1 \mathrm{D}}\left|z_{i}+z_{j}\right|\right)\right] \hat{\sigma}_{e g}^{i} \hat{\sigma}_{g e}^{j}
\end{aligned}
$$

and $\Gamma_{i, j}=\Gamma_{1 \mathrm{D}}\left[\cos \left(k_{1 \mathrm{D}}\left|z_{j}-z_{i}\right|\right)-\cos \left(k_{1 \mathrm{D}}\left|z_{j}+z_{i}\right|\right)\right]$.

While Eqs. (2) and (4) have now eliminated the infinite Hilbert space of photons, an interesting new feature emerges: dissipation. In particular, MBL is usually formulated with respect to closed systems. Although a dissipative environment or coupling to a bath might generically be expected to destroy MBL, it can in some cases lead to nontrivial behavior, such as if the bath itself is many-body localized or contains too few degrees of freedom $[11,18,58]$. Here we find an interesting behavior that appears physically distinct from the aforementioned examples. In particular, although the dissipative part of the master equation a priori has the same strength $\left(\sim \Gamma_{1 \mathrm{D}}\right)$ as the coherent interactions, the bulk region of a many-body system behaves as if it is increasingly closed as it becomes further in distance from a boundary, as suppressed transport makes the emission of a photon past the system edges improbable and then makes the notion of MBL self-consistent. These ideas can be better clarified by returning to the simpler
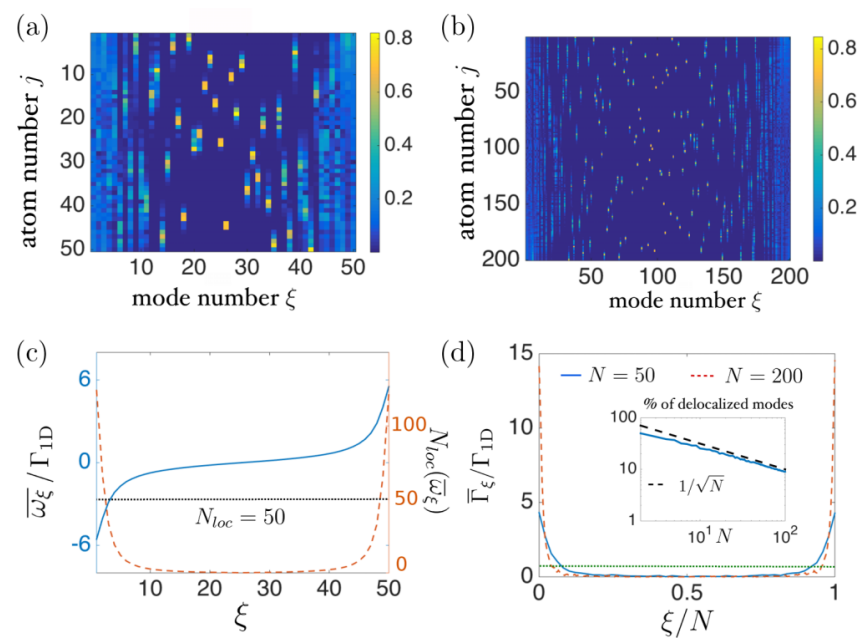

FIG. 2. Spatial profile of the single-excitation eigenmodes $\left|c_{j}^{\xi}\right|$ of the effective Hamiltonian $\mathcal{H}_{1 \mathrm{D}}$, ordered with respect to the real part of their eigenvalue, for two different system sizes: $N=50$ (a) and $N=200$ (b). These eigenmodes have been calculated for a single disorder realization, and are indexed from lowest $(\xi=1)$ to highest $(\xi=N)$ frequency. (c) Average value over a large number of disorder realizations of $\omega_{\xi} / \Gamma_{1 \mathrm{D}}$ (solid blue line) as a function of $\xi$, together with the associated Anderson localization length $N_{\text {loc }}\left(\bar{\omega}_{\xi}\right)$ (dashed orange line) in units of number of atoms. This plot is calculated for $N=50$ atoms. For reference, we indicate where the localization length corresponds to the system size by the dotted black line. (d) Average value over a large number of disorder realizations of the normalized decay rate $\Gamma_{\xi} / \Gamma_{1 \mathrm{D}}$ of eigenmodes, as a function of $\xi / N$, for two different system sizes $N=50$ (solid blue line) and $N=200$ (dashed orange line). A large fraction of modes have a decay rate smaller than $\Gamma_{1 \mathrm{D}} / 2$ (dotted green line). Numerically, the percentage of modes with decay rate larger than $\Gamma_{1 \mathrm{D}} / 2$ decreases approximately as $\sim 1 / \sqrt{N}$ (inset).

problem of Anderson localization, but now studied from the spin model perspective.

\section{A. Disorder in the single-photon limit}

The key single-excitation properties are encoded in the $N$ single-excitation eigenmodes and eigenvalues of the nonHermitian Hamiltonian $\mathcal{H}_{1 \mathrm{D}}$. The eigenvalues themselves are complex, with the real and imaginary parts accounting for the shift in resonance frequency $\omega_{\xi}$ of the collective mode with respect to the bare atomic frequency, and half of the collective decay rate $\Gamma_{\xi} / 2$, respectively.

As a concrete example, we first take one single realization of disorder and sort the eigenstates in increasing order of their resonance frequencies, labeled by $1 \leqslant \xi \leqslant N$. Denoting the wave functions by $\left|\psi_{\xi}\right\rangle=\sum_{j} c_{j}^{\xi} \hat{\sigma}_{e g}^{j}|g\rangle^{\otimes N}$, in Figs. 2(a) and 2(b) we plot $\left|c_{j}^{\xi}\right|$, the square root of the probability for atom $j$ to be excited, for the atom numbers of $N=50$ and $N=200$, respectively. For all numerical calculations, we take an average atomic spacing of $d=2.7 \pi / k_{1 \mathrm{D}}$ (to be in the dilute regime) and full disorder. We see that states in the middle of the spectrum $(\xi \sim N / 2)$ are localized, while states at the edges of the spectrum $(\xi \sim 1$ and $\xi \sim N)$ are extended. Furthermore, in Fig. 2(c), we fix the atom number 

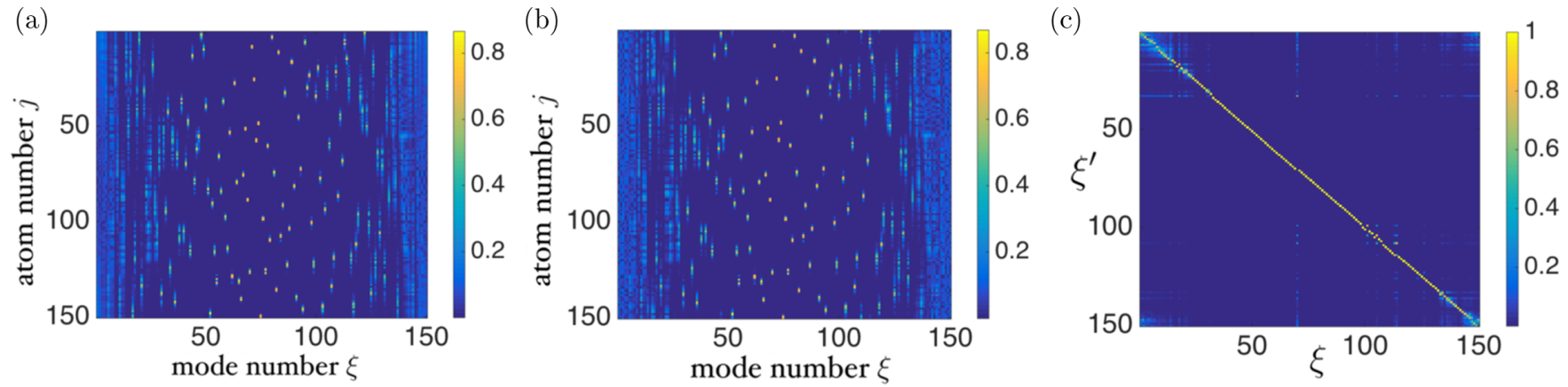

FIG. 3. A representative plot of the excitation amplitude $\left|c_{j}^{\xi}\right|$ versus atom number $j$ and eigenmode index $\xi$, for a single disorder realization and within the single-excitation manifold. The excitation amplitudes are plotted for (a) the effective non-Hermitian Hamiltonian $\mathcal{H}_{1 \mathrm{D}}$ and (b) its Hermitian component $\mathcal{H}_{\text {hermi }}$. (c) Magnitude of the dot product between the eigenmodes of the two Hamiltonians: $D\left(\xi, \xi^{\prime}\right)=\left|\left\langle\psi_{\xi}^{(1 D)} \mid \psi_{\xi^{\prime}}^{(\text {hermi })}\right\rangle\right|$. All of these plots were generated for a system of $N=150$ atoms.

$N=50$, and plot the average value of the resonance frequency $\bar{\omega}_{\xi} / \Gamma_{1 \mathrm{D}}$ versus $\xi$ over a large number of disorder realizations (blue solid curve). We simultaneously plot (orange dashed curve) the previously established Anderson localization length $N_{\text {loc }}\left(\bar{\omega}_{\xi}\right)$ corresponding to these frequencies. Comparing with Fig. 2(a), we see that the transition from localized to extended eigenstates occurs when $\left|\bar{\omega}_{\xi}\right|$ is detuned enough that the localization length $N_{\mathrm{loc}}\left(\bar{\omega}_{\xi}\right)>N$ exceeds the number of atoms inside the chain. In particular, the existence of extended states of the spin model (such as found in Refs. [59-61]) does not contradict the fact that Anderson localization always exists in the 1D disordered system. Specifically, for any detuning $\Delta$ there always exists some sufficiently large $N>N_{\text {loc }}$ where one will eventually observe Anderson localization.

In Fig. 2(d), we plot the average value over a large number of disorder realizations of the decay rate $\bar{\Gamma}_{\xi} / \Gamma_{1 \mathrm{D}}$, normalized by the single-atom decay rate, as a function of $\xi / N$ for two different system sizes $N=50$ and $N=200$. We see that the extended modes in the edges of the spectrum have a large decay rate, while the localized modes in the middle have an exponentially small decay rate. This confirms that localized modes, with populations away from the boundaries, cannot efficiently decay by spontaneous emission. Furthermore, as $N$ increases, we find that the percentage of delocalized modes [here quantified by the percentage of modes with a decay rate greater than $\Gamma_{1 \mathrm{D}} / 2$, above the green dotted line in Fig. 2(d)] decreases approximately as $\sim 1 / \sqrt{N}$, as shown in the inset.

While this analysis was restricted to the single-excitation manifold, it naturally also follows that for our particular model, the existence of MBL cannot be inferred purely by looking for the localized nature of eigenstates of the spin model with large number of excitations. In particular, a study along those lines would have to determine whether an apparently delocalized state might become localized as the system size is increased. As that is difficult within numerical capabilities, we must demonstrate the existence of MBL using other measures. Separately, we note that while localized and extended single-excitation eigenstates can be identified by spectral filtering (i.e., looking at eigenstates at the center and edges of the spectrum, respectively), this procedure cannot be extended to more excitations. For example, assuming that interactions can be considered as a perturbation, two single-excitation extended eigenstates with energies $\pm \omega$ could combine to give an extended two-excitation eigenstate with approximately zero energy.

\section{B. Localization as a transition to Hermitianity}

If Anderson (or many-body) localized states experience exponentially small decay rates versus distance of excitations from boundaries, then the dissipative component of Eq. (2) should have negligible effect, and such states should essentially be governed only by the Hermitian component $\mathcal{H}_{\text {hermi }}=$ $\left(\mathcal{H}_{1 \mathrm{D}}+\mathcal{H}_{1 \mathrm{D}}^{\dagger}\right) / 2$ of the effective Hamiltonian. This Hermitian component reads:

$$
\mathcal{H}_{\text {hermi }}=\frac{\Gamma_{1 \mathrm{D}}}{2} \sum_{i, j} \sin \left(k_{1 \mathrm{D}}\left|z_{i}-z_{j}\right|\right) \hat{\sigma}_{g e}^{i} \hat{\sigma}_{e g}^{j} .
$$

We can again easily confirm this in the single-excitation limit. Specifically, for a single disorder realization and $N=$ 150 , we calculate the eigenstate amplitudes $\left|c_{j}^{\xi}\right|$ for the nonHermitian and Hermitian Hamiltonians, which we plot in Figs. 3(a) and 3(b), respectively. Then, in Fig. 3(c), we plot the overlap $D\left(\xi, \xi^{\prime}\right)=\left|\left\langle\psi_{\xi}^{(1 \mathrm{D})} \mid \psi_{\xi^{\prime}}^{(\text {hermi })}\right\rangle\right|$ between the nonHermitian and Hermitian eigenstates. We find that this matrix is nearly the identity, particularly in the middle of the spectrum where the modes are well localized [62].

This suggests that we can establish the existence of an MBL in two complementary ways. First, we can take the Hermitian Hamiltonian $\mathcal{H}_{\text {hermi }}$, and look for well-established mathematical signatures for closed systems, such as logarithmic growth of entanglement entropy following a quench. Then, assuming that the existence of MBL is not affected by losses for the reasons above, we can simultaneously look for realistic observables in the physical, open system.

\section{INTRODUCTION TO CONVENTIONAL MBL}

The key properties of MBL can be understood from a "canonical" Hamiltonian hypothesized for all MBL systems 


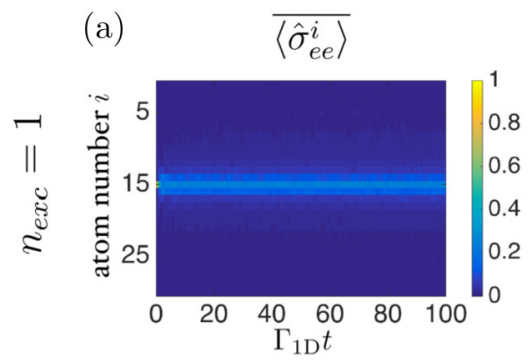

(b) $\quad\left\langle\hat{\sigma}_{e e}^{i}\right\rangle$
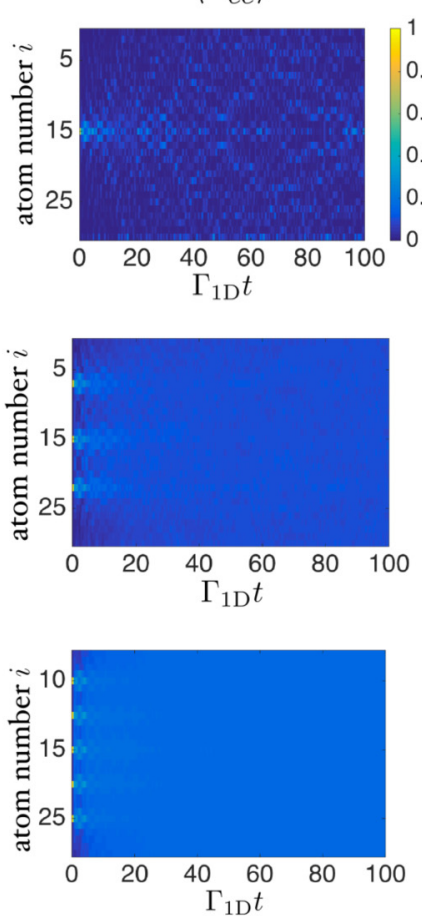

(c)
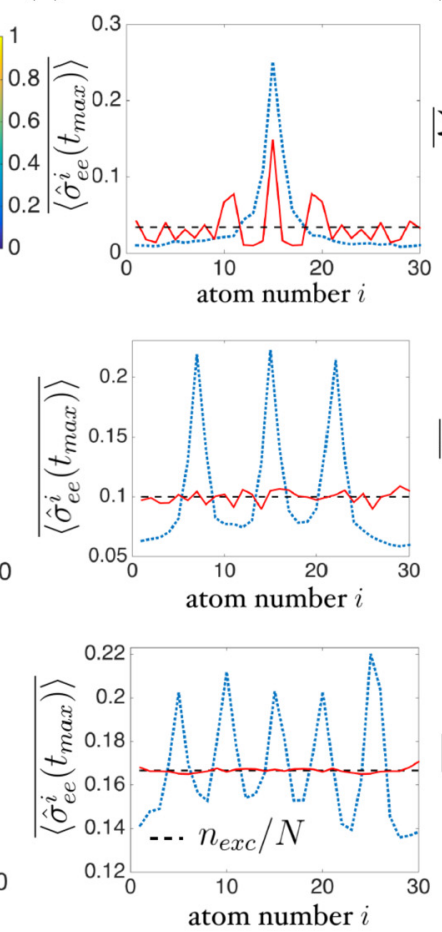

(d)
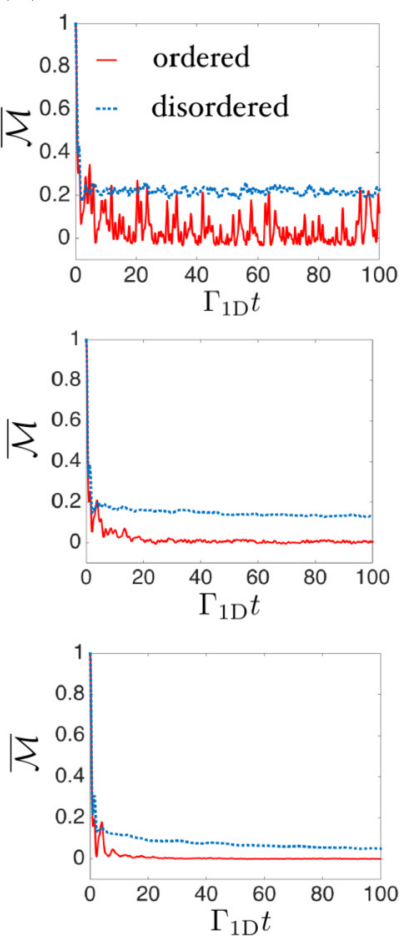

FIG. 4. Transport properties of $N=30$ atoms in the Hermitian waveguide, averaged over disorder configurations. Each line corresponds to a different number of excitations $\left(n_{\mathrm{exc}}=1,3,5\right)$, initialized in a product state. In (a) and (b), we plot the time-dependent, site-resolved excited-state population $\left\langle\hat{\sigma}_{e e}^{i}(t)\right\rangle$, for disordered and ordered systems, respectively. (c) Site-resolved population $\left\langle\hat{\sigma}_{e e}^{i}\left(t_{\max }\right)\right\rangle$ evaluated at the final simulation time $t_{\max }=100 / \Gamma_{1 \mathrm{D}}$, for both disordered (blue dotted line) and ordered systems (solid red line). As a guide to the eye, we plot $n_{\text {exc }} / N$ (dashed line), the population if the initial excitations were to become uniformly distributed. (d) Time-dependent memory parameter $\overline{\mathcal{M}}(t)$ for both disordered (blue dotted line) and ordered systems (solid red line).

$[63,64]$. For spin systems like ours, it reads

$$
\mathcal{H}_{\mathrm{LIOM}}=\sum_{i} \omega_{i} \hat{\tau}_{z}^{i}+\sum_{i, j} J_{i, j} \hat{\tau}_{z}^{i} \hat{\tau}_{z}^{j}+\sum_{i, j, k} G_{i, j, k} \hat{\tau}_{z}^{i} \hat{\tau}_{z}^{j} \hat{\tau}_{z}^{k}+\ldots
$$

Here the $\hat{\tau}_{z}^{i}$ operators are so-called local integrals of motion, pseudo- $\hat{\sigma}_{z}$ operators that are quasilocal in space.

Because $\mathcal{H}_{\text {LIOM }}$ only involves products of $\hat{\tau}_{z}$ operators, each $\hat{\tau}_{z}^{j}$ commutes with the Hamiltonian and thus their occupancies are conserved quantities. Furthermore, as these operators only have support on a few sites, there will be no transport of energy, and an MBL system prepared initially out of equilibrium will never thermalize.

The interacting terms (e.g., $J_{i, j}$ for two-body interactions) have exponentially decreasing amplitude with the distance between modes [64-66]. The interactions differentiate MBL from Anderson localization, and cause each local integral of motion $\hat{\tau}_{z}^{i}$ to acquire different phases in evolution depending on the occupancy of other $\hat{\tau}_{z}^{j}$. For closed systems, this results in a dephasing for any local subsystem, due to the gradual entanglement of these degrees of freedom with others further away. Likewise, starting from a product state in the physical basis, these interactions cause a subsystem $\hat{\rho}_{A}$ consisting of half the entire system to exhibit a logarithmic growth of entanglement entropy $S(t)=-\operatorname{Tr}\left\{\hat{\rho}_{A}(t) \log \left[\hat{\rho}_{A}(t)\right]\right\}$ in time in the MBL phase [67]. As a comparison, systems that do not exhibit MBL experience a ballistic growth of entanglement entropy (linear with time).

\section{MANY-BODY LOCALIZATION IN THE HERMITIAN WAVEGUIDE MODEL}

In this section, we study the Hermitian component $\mathcal{H}_{\text {hermi }}$ of the effective Hamiltonian, looking at transport and entanglement entropy. Specifically, we consider initial states consisting of product states of $n_{\text {exc }}$ excitations, $\left|\psi_{\text {init }}\right\rangle=$ $\prod_{j \in \mathcal{E}_{\text {init }}} \hat{t}_{e g}^{j}|g\rangle^{\otimes N}$ with $\mathcal{E}_{\text {init }}$ being the set of indices of the initially excited atoms. Enforcing a fixed number of excitations enables us to study modestly larger system sizes, by throwing out the Hilbert subspace with higher excitation numbers.

We first consider transport, in a system of $N=30$ atoms. In Fig. 4(a), we show the time-dependent excited-state population $\left\langle\hat{\sigma}_{e e}^{i}(t)\right\rangle$ per site, averaged over $\sim 20-100$ disordered configurations, for $n_{\text {exc }}=\{1,3,5\}$ roughly equidistantly spaced excitations. In comparison, in Fig. 4(b), we show the evolution for the same initial states, but in an ordered chain of lattice constant $d=2.7 \pi / k_{1 \mathrm{D}}$. In Fig. 4(c) we plot the site-dependent population $\left\langle\hat{\sigma}_{e e}^{i}\left(t_{\max }\right)\right\rangle$ for the final time of the simulation, $t_{\max }=100 / \Gamma_{1 \mathrm{D}}$, both for the disordered (blue dotted line) and ordered cases (red solid line). In the ordered system, the population rapidly becomes distributed evenly among all the spins, so that $\left\langle\hat{\sigma}_{e e}^{i}\left(t_{\max }\right)\right\rangle \approx n_{\text {exc }} / N$ (dashed black line). (The only exception is for $n_{\text {exc }}=1$, where the single excitation evolves according to a well-defined band dispersion relation.) In contrast, in the presence of disorder, the system retains a clear memory. 


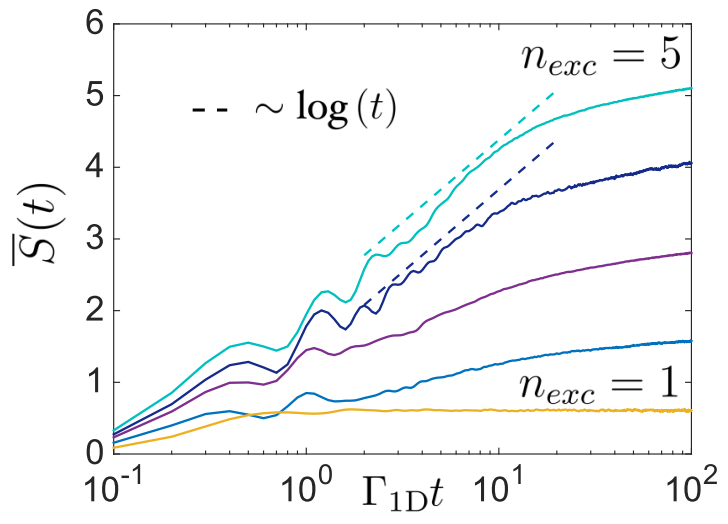

FIG. 5. Disorder-averaged half-chain entanglement entropy $\bar{S}(t)$ as a function of time, for different number of atomic excitations initially prepared in a product state, in the Hermitian waveguide. The simulations are for $N=30$ atoms, and the curves from bottom to top correspond to $1 \leqslant n_{\text {exc }} \leqslant 5$ excitations. Logarithmic scalings (dashed lines) are shown as a guide to the eye.

This can be quantified by defining an observable that captures the memory of the initial state:

$$
\mathcal{M}(t)=\left[\frac{\sum_{i \in \mathcal{E}_{\mathrm{init}}}\left\langle\hat{\sigma}_{e e}^{i}(t)\right\rangle}{n_{\mathrm{exc}}}-\frac{n_{\mathrm{exc}}}{N}\right] /\left(1-\frac{n_{\mathrm{exc}}}{N}\right) .
$$

This quantity would be equal to 1 in the case of an initial population distribution conserved through time, and to 0 in the case of an equally shared population between all atoms. In Fig. 4(d), we plot the disorder-averaged $\overline{\mathcal{M}}(t)$ and observe a clear memory of the initial state (or, equivalently, an absence of transport at long times) in all the cases with disorder.

In Fig. 5 we study the evolution of the half chain entanglement entropy $S(t)$ in time, for the same disordered system and initial conditions. A clear region of logarithmic growth is observed when the number of excitations is large enough (up to the largest number $n_{\text {exc }}=5$ we can simulate in a system of $N=30$ atoms), before saturating due to the limited system size. Figures 4 and 5 provide evidence that the Hermitian system, as defined by $\mathcal{H}_{\text {hermi }}$, exhibits a many-body localized phase up to an excitation density of at least $\sim 1 / 6$.

\section{A. Delocalization transition}

As discussed in Sec. II, based on qualitative arguments, we expect a disordered waveguide QED system to exhibit an MBL phase up to an excitation density of $\rho_{e e}=n_{\operatorname{exc}} / N=1 / 2$ in the thermodynamic limit. However, we also expect that the localization length $N_{\text {loc }}\left(\Delta, \rho_{e e}\right)$ should gradually increase as the excitation density increases, eventually diverging as $\rho_{e e} \rightarrow 1 / 2$. Thus, for a finite system of moderate size as can be simulated, this would lead to a smooth crossover to delocalization as $n_{\text {exc }}$ is increased.
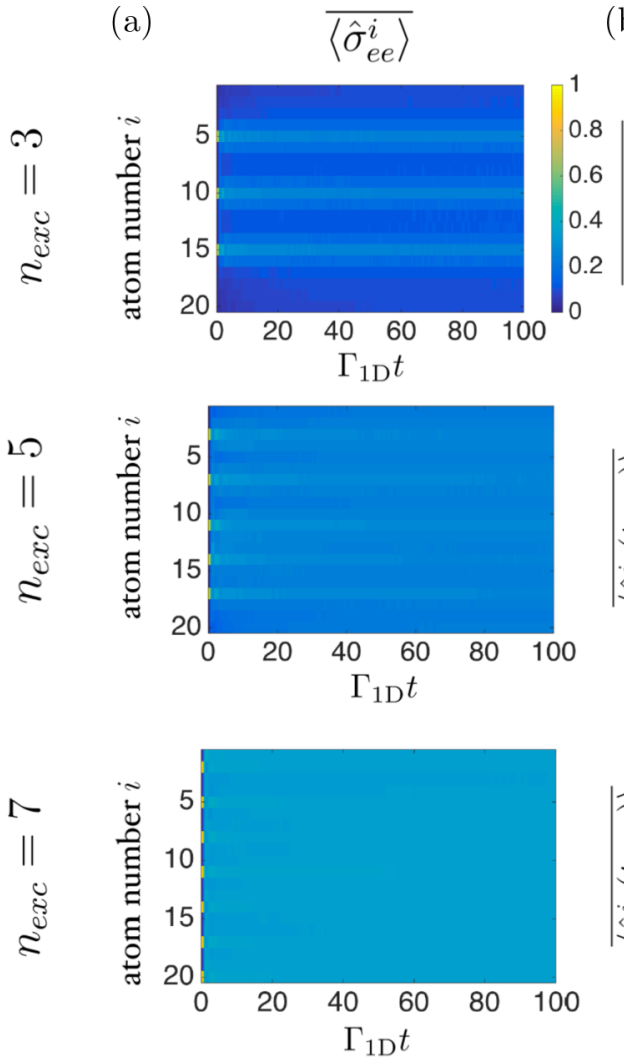

(b)
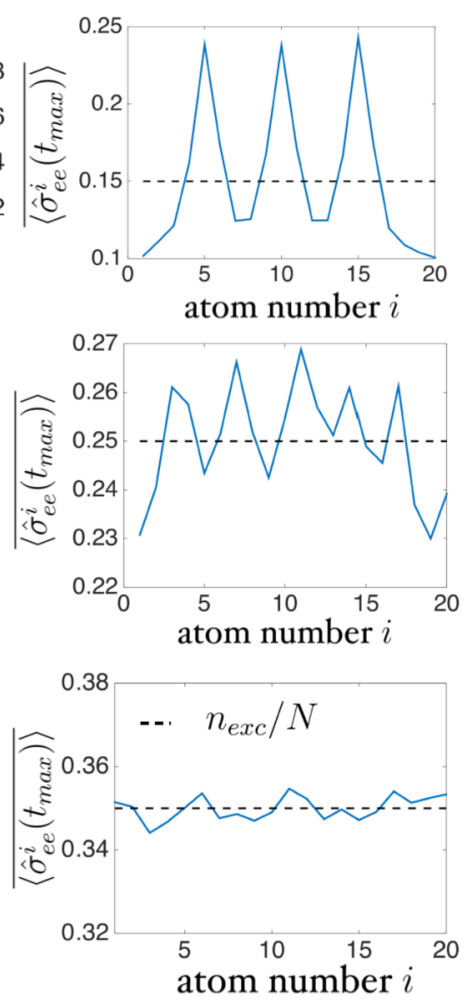

(c)
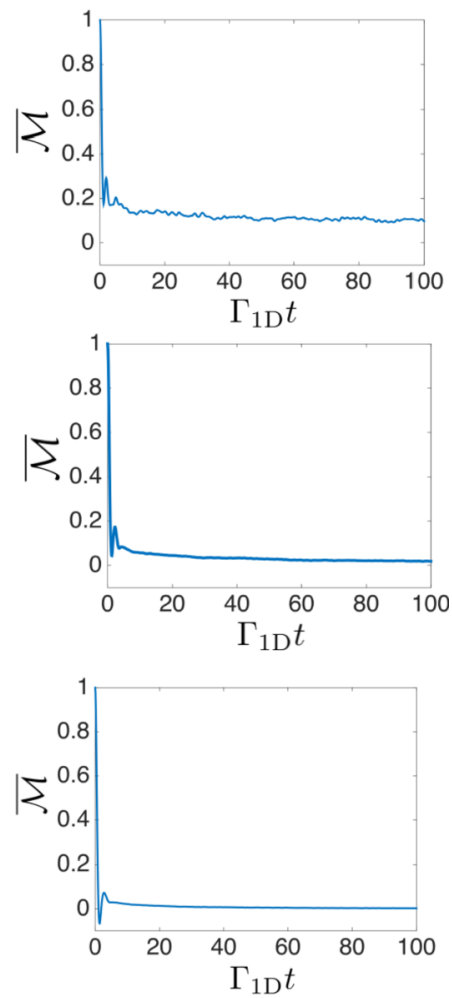

FIG. 6. Transport properties of $N=20$ atoms in the Hermitian waveguide, averaged over disorder configurations. Each line corresponds to a different number of excitations $\left(n_{\text {exc }}=3,5,7\right)$, initialized in a product state. (a) Time-dependent, site-resolved excited-state population $\left\langle\hat{\sigma}_{e e}^{i}(t)\right\rangle$. (b) Site-resolved excited-state population at the final simulation time, $t_{\max }=100 / \Gamma_{1 \mathrm{D}}$. As a guide to the eye, we plot $n_{\mathrm{exc}} / N$ (dashed line) to indicate the population if the excitations become equally distributed. (c) Time-dependent memory parameter $\overline{\mathcal{M}}(t)$. 


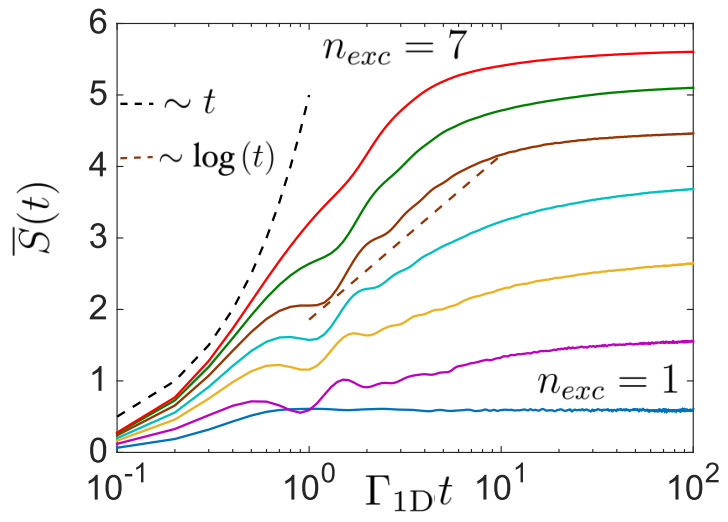

FIG. 7. Disorder-averaged half-chain entanglement entropy $\bar{S}(t)$ as a function of time, for different number of atomic excitations initially prepared in a product state, in the Hermitian waveguide. The simulations are for $N=20$ atoms, and the curves from bottom to top correspond to $1 \leqslant n_{\mathrm{exc}} \leqslant 7$ excitations. Linear and logarithmic scalings (dashed curves) are shown as a guide to the eye.

To observe this, we consider a smaller chain of $N=20$, and repeat the same calculations as before for the Hermitian waveguide, but now for $1 \leqslant n_{\mathrm{exc}} \leqslant 7$ to reach a higher excitation density. In Fig. 6(a) we plot the transport properties for $n_{\mathrm{exc}}=3,5$ and 7 excitations. It can be seen that the memory of the initial state becomes negligible already for $n_{\mathrm{exc}}=5$, and essentially vanishes for $n_{\mathrm{exc}}=7$, as the initial population is eventually equally shared among all atoms in the waveguide. In Fig. 7 we plot the half-chain entanglement entropy, and see a smooth transition toward ballistic growth as the number of excitations exceeds $n_{\text {exc }} \gtrsim 4-5$.

While a delocalization transition that occurs at a lower density of excitations, $n_{\text {exc }} / N \sim 1 / 4$, cannot be ruled out from these numerics, the results are also consistent with our previous hypothesis of a smooth crossover. In particular, assuming that the dynamics have a characteristic bandwidth of $\sim \Gamma_{1 \mathrm{D}}$, one finds a heuristic many-body localization length $N_{\text {loc }}(\Delta=$ $\left.\Gamma_{1 \mathrm{D}}, \rho_{e e}\right)$ that exceeds the system size of $N=20$ for $n_{\mathrm{exc}} \gtrsim 4$.

\section{MBL IN THE OPEN SYSTEM}

\section{A. Quantum transport}

We now investigate practical signatures of MBL in the physical, open system. Anticipating that regions within the excitation density-dependent localization length $\sim N_{\text {loc }}\left(\Delta, \rho_{e e}\right)$ of the boundaries are strongly affected by dissipation, we reduce their relative contribution in numerics by going to the open half-waveguide, as described by Eq. (4), which only has one radiating boundary located to the right.

We begin by studying transport in the open system. Based on the intuition that the system will resemble a closed system (and thus exhibit stronger features of MBL) provided that excitations remain far from the boundary, we slightly alter the initial conditions compared to Sec. V, and take initial states consisting of $n_{\text {exc }}$ excitations all located on the left half of the chain. To eliminate any bias originating from a specific choice of location within the left half, we consider equal superpositions of all possible basis states, with each basis state multiplied by a random phase. We have checked, however, that other choices do not affect the final conclusions. Numerically, we directly solve for the master equation dynamics of Eq. (4), for $n_{\mathrm{exc}} \leqslant 2$, and by a quantum jump approach for higher excitation number, with a minimum of 150 trajectories for each configuration, and with at least 50 random configurations in all cases to calculate disorder-averaged observables.

We then define the time-dependent imbalance $\mathcal{M}_{\text {half }}(t)$ between the left and right halves,

$$
\mathcal{M}_{\text {half }}(t)=\frac{1}{P_{e}(t)}\left[\sum_{i<N / 2+1}\left\langle\hat{\sigma}_{e e}^{i}(t)\right\rangle-\sum_{i>N / 2}\left\langle\hat{\sigma}_{e e}^{i}(t)\right\rangle\right],
$$

where $P_{e}(t)=\sum_{i}\left\langle\hat{\sigma}_{e e}^{i}(t)\right\rangle$ is the total excited population that now decays in time. In Fig. 8(a) we plot the disorder averaged, time-dependent imbalance for various initial excitation numbers $n_{\text {exc }}=1,2,4,6$ and for different system sizes (indicated by different colors). Alongside the imbalance, we plot in Fig. 8(b) the total population $P_{e}(t)$.

Beginning with a low density of excitations $\left(n_{\mathrm{exc}}=1,2\right)$ in the disordered system (solid lines), we see that both transport and dissipation are strongly suppressed, with the asymptotic behavior apparently trending toward perfect suppression, $\mathcal{M}_{\text {half }} \rightarrow 1$ and $P_{e} \rightarrow n_{\text {exc }}$, as $N \rightarrow \infty$. This confirms the increasingly closed nature of the system. Similar trends also are observed for a higher number of excitations, $n_{\mathrm{exc}}=4$. Here, however, the localization length $N_{\mathrm{loc}}\left(\Delta=\Gamma_{1 \mathrm{D}}, \rho_{e e}\right)$ (again assuming a characteristic bandwidth $\sim \Gamma_{1 \mathrm{D}}$ for dynamics) becomes comparable to the system sizes simulated, and dissipation is no longer negligible. Interestingly, one sees that the imbalance does not monotonically decay, but rather reaches a minimum over a short timescale, while partially recovering at later times. This behavior is more prominent for smaller $N$, due to the higher initial excitation density. Physically, part of the initial population on the left experiences transport and quickly propagates toward the right boundary of the chain (decreasing the imbalance), where it can be dissipated. The lowering of density on the left half subsequently suppresses the transport, and the system dynamically enters into an MBL phase, while the imbalance partially recovers as the remaining excitations on the right half then slowly but preferentially dissipate away. Given this observation, we are then motivated to investigate even higher initial densities $\left(n_{\mathrm{exc}}=6\right.$ and $N=$ $16)$, where now the localization length $N_{\text {loc }}\left(\Delta=\Gamma_{1 \mathrm{D}}, \rho_{e e}=\right.$ $3 / 8) \approx 65 \gg N$ far exceeds the system size. Despite clearly starting in a transport and dissipation-allowed phase, the same dynamical behavior is clearly and even more prominently observed.

We now return to the hypothesized phase diagram of Fig. 1(b), which we initially considered to be for a system in the thermodynamic limit. Considering that any physical system will consist of finite atom number, we propose that its dynamical behavior, if it begins in the delocalized phase, will consist of transport-facilitated dissipation, until it reaches an MBL phase, as illustrated by the arrow showing evolution in time. Furthermore, this dissipation process should be quite rapid and dominate the initial dynamics, due to the lack of subradiant states (with decay rate $\ll \Gamma_{1 D}$ ) for excitation densities $n_{\text {exc }} / N \gtrsim 1 / 2$, and by the fact that the average dissipation rate of eigenstates of the non-Hermitian Hamiltonian for such 


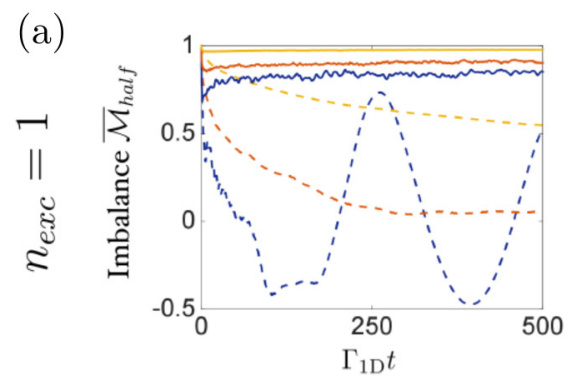

(b)
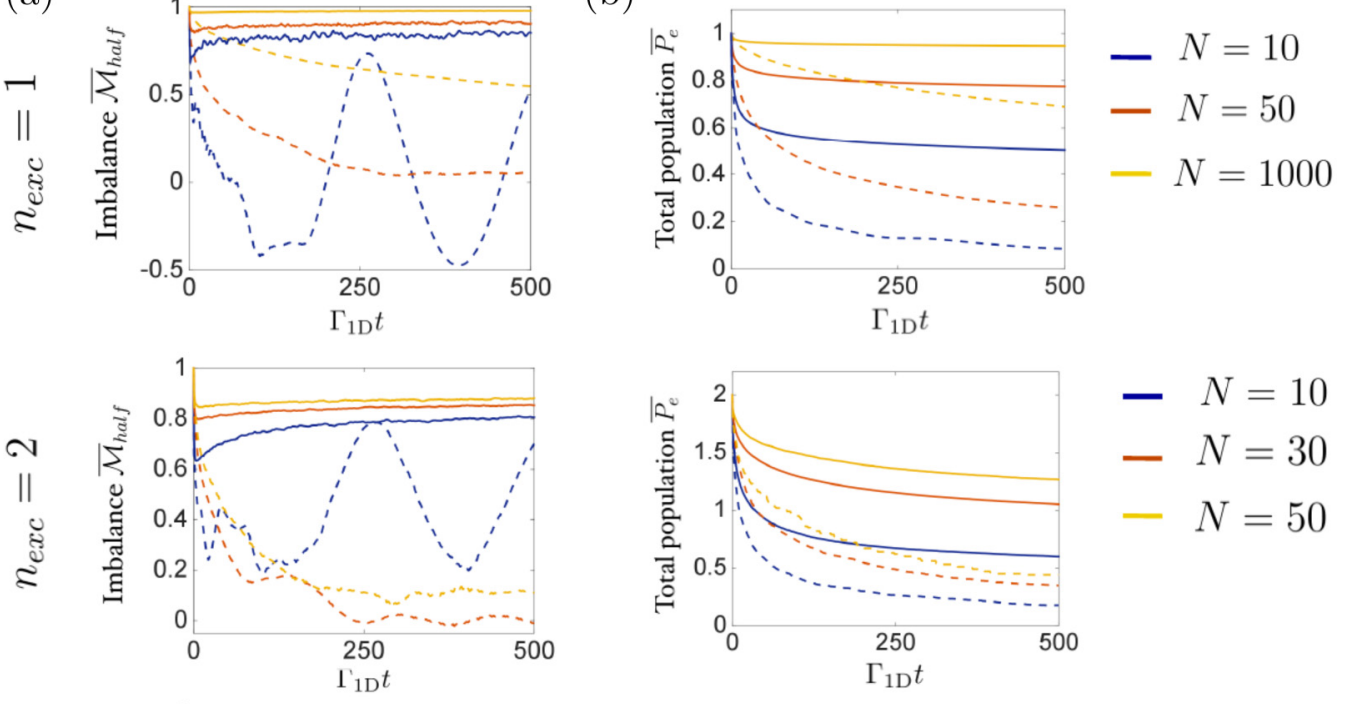

$-N=10$
$-\quad N=30$
$-N=50$
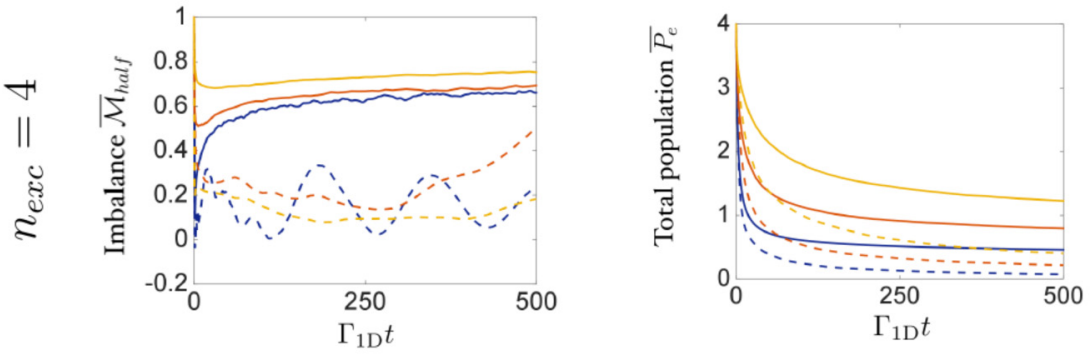

$$
\begin{aligned}
& -N=8 \\
& -N=14 \\
& -N=26
\end{aligned}
$$
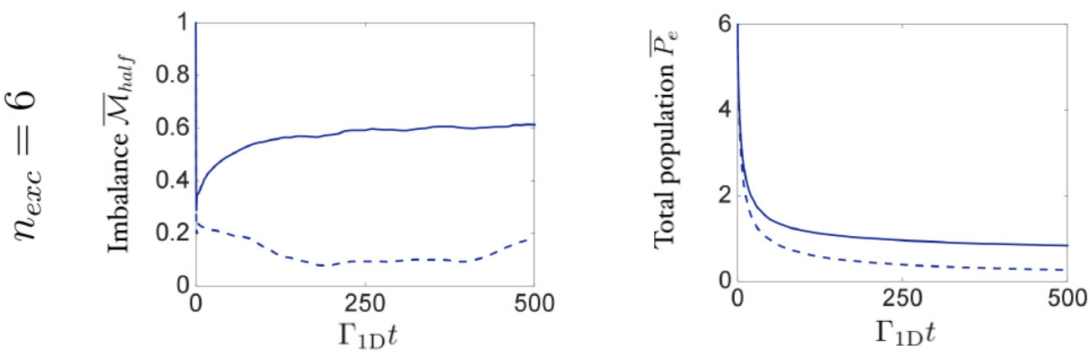

$-N=16$

FIG. 8. Time-dependent, disorder-averaged population imbalance $\overline{\mathcal{M}}_{\text {half }}(t)$ (a) and total population $\bar{P}_{e}(t)$ (b) for the open half-waveguide, for $n_{\text {exc }}=1,2,4,6$ initial excitations, and for various system sizes $N$ (indicated by different colors). We plot these quantities both for the disordered (solid curves) and ordered (dashed) systems.

high excitation densities must grow extensively with system size, $\sim N \Gamma_{1 \mathrm{D}}$.

Finally, for comparison, we plot with dashed curves the same observables for all the system sizes and number of excitations previously considered, but for an ordered system. It should be noted that the total population in the ordered system also decays slowly for long times, albeit faster than the disordered case. This can be attributed to the large number of single and multiexcitation subradiant states in a $1 \mathrm{D}$ waveguide at low excitation density [54]. In the case of a full waveguide (dissipation at both boundaries) and sufficient number of excitations, it has previously been shown that this leads to a power-law decay for ordered systems at long times [6].

\section{B. Quantum revivals}

As entanglement entropy is difficult to measure in a closed system of large size, and has no immediate analog in an open system, capturing some essence of the slow entanglement en- tropy growth is generally one of the most challenging aspects of experimentally studying MBL [68]. When the local integral of motion closely resembles the physical spin, it has been theoretically proposed that the slow growth of entanglement can be probed by generalized spin echo techniques [69]. In our system, however, we find that this technique is ineffective, as localized modes have a non-negligible extent over at least a few sites (see the single-excitation dynamics in Fig. 4, for example). Physically, this is because suppressing the emission of light by one excited atom arises from the excitation of at least the neighboring atoms and their destructive interference in radiation.

We thus adopt an alternative proposal by Vasseur et al. [70], which is readily implementable within waveguide QED. In particular, we consider the addition of an extra ancilla atom $A$ that does not directly couple or radiate into the waveguide, but is allowed to coherently exchange excitations with the waveguide atom with index $n_{c}$ [see Fig. 9(a)]. The waveguide atoms thus serve as an exotic bath for the ancilla. The corresponding 

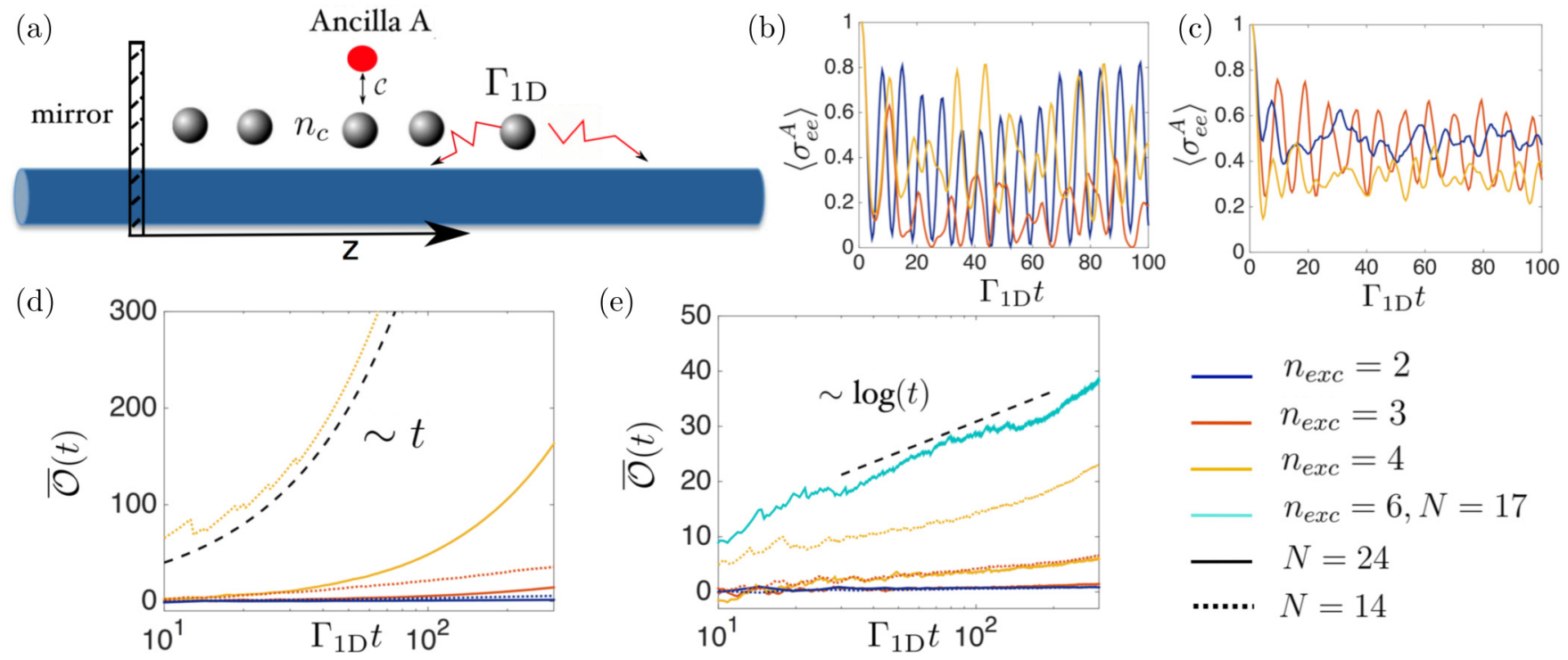

FIG. 9. (a) Scheme of the quantum revival setup to measure the interacting degrees of freedom in the open half-waveguide. An additional ancilla $A$ is introduced, which does not couple or radiate into the waveguide, but couples to and can exchange excitations with the waveguide atom $n_{c}$. The time-dependent excited population of the ancilla is monitored to record the number of revivals $N_{\text {rev }}$ within a certain time window $[0, t]$. [(b) and (c)] Ancilla population versus time, for three typical disorder realizations for a Hermitian half-waveguide. Here initially the ancilla is excited, while the waveguide atoms contain no excitations (b) and 2 excitations located at positions $\left|e_{1}, e_{4}\right\rangle$ (c). The total system size (ancilla + atoms) is $N=14$. Disorder-averaged $\mathcal{O}(t)$ for both Hermitian (d) and open (e) disordered systems of size $N=14$ (dotted curves), $N=17$ (light blue curve), and $N=24$ (solid curves) with varying number of excitations $\left(n_{\text {exc }}=2,3,4,6\right)$ indicated by different colors. As a guide to the eye, we show a linear evolution in (d) and a logarithmic evolution in (e) in black dashed curves.

Hamiltonian for this system (studied for the half-waveguide) is

$$
H=\mathcal{H}_{\text {half }}+\mathcal{H}_{c},
$$

with the non-Hermitian Hamiltonian $\mathcal{H}_{\text {half }}$ given in Eq. (5) and $\mathcal{H}_{c}=\mathcal{C}\left(\hat{\sigma}_{e g}^{A} \hat{\sigma}_{g e}^{n_{c}}+\right.$ H.c. $)$ coupling the ancilla to atom $n_{c}$ in the chain. In the following we use $\mathcal{C}=\Gamma_{1 \mathrm{D}} / 2$ and we couple the ancilla to the atom number $n_{c}=3$ in the chain of $N-1$ atoms.

If the system was governed purely by $\mathcal{H}_{c}$, then an ancilla initially excited would indefinitely and reversibly exchange its excitation with atom $n_{c}$. In the Anderson localized regime, where the waveguide interactions are turned on but all waveguide atoms are initially unexcited, the ancilla couples to a few localized modes (those whose support contain atom $n_{c}$ ). This leads to a more complex structure of collapse and revival dynamics in the ancilla population over time, as illustrated in Fig. 9(b) (calculated here for the Hermitian half-waveguide, with no dissipation). Although the details of the revivals depend on the disorder configuration, since the number of localized modes to which the ancilla couples is finite and fixed, the revivals would continue with the same frequency and amplitude indefinitely in the absence of dissipation, and for an increasingly long time with increasing system size in the presence of dissipation. In contrast, in the MBL phase where multiple waveguide atoms are initially excited:

$$
\left|\psi_{\text {init }}\right\rangle=\left|e_{A}\right\rangle \otimes\left|\psi_{\mathrm{wg}}\right\rangle,
$$

with $\left|\psi_{\mathrm{wg}}\right\rangle$ containing $n_{\mathrm{exc}}-1$ excitations, interactions between excitations effectively cause the ancilla to couple to an increasing number of degrees of freedom in time, which makes revivals less likely. This is illustrated in Fig. 9(c), for the case of $n_{\mathrm{exc}}-1=2$ excitations initialized in the Hermitian half-waveguide as well, at positions $\left|e_{1}, e_{4}\right\rangle$.

For closed systems, the revival rate should approximately be inversely proportional to the effective size of the Hilbert space to which the ancilla is coupled. We can thus numerically study the revival rate $R(t)=N_{\mathrm{rev}}(t) / t$ (see Appendix B for how the number of revivals $N_{\mathrm{rev}}(t)$ in the time window $[0, t]$ is determined). To do this, we again directly solve the master equation for low excitation numbers $n_{\mathrm{exc}}=1,2$, and by the quantum jump formalism for higher numbers (with at least 500 trajectories for each configuration). We perform averages over at least 50 configurations for each choice of $n_{\mathrm{exc}}, N$, and the initial waveguide state is chosen to be in an equal superposition of all basis states with $n_{\text {exc }}-1$ excitations, multiplied by random phases.

To quantify the evolution of the average number of degrees of freedom $\overline{\mathcal{N}}(t)$ to which the ancilla is coupled by interactions, we take the ansatz that

$$
\bar{R}(t) \approx \frac{\alpha}{\overline{\mathcal{N}}(t)},
$$

where $\alpha$ is a proportionality constant. We further split $\overline{\mathcal{N}}(t)=$ $\overline{\mathcal{N}}_{0}(t)+\overline{\mathcal{N}}_{\text {int }}(t)$ with $\overline{\mathcal{N}}_{0}(t)$ the average number of degrees of freedom that interacts with the ancilla without interactions inside the waveguide and $\overline{\mathcal{N}}_{\text {int }}(t)$ the average number of degrees of freedom that comes from interactions between the different qubits. $\overline{\mathcal{N}}_{\text {int }}(t)$ should grow logarithmically in time for closed, large MBL systems and linearly in time for delocalized systems, while $\overline{\mathcal{N}_{0}}(t)$ saturates to a constant value that depends on the localization length $N_{\text {loc }}$.

Thus, using the unloaded waveguide as a reference to obtain $\bar{R}_{0}(t)$, we can extract from the revival rates the average 
number of interacting degrees of freedom (up to a proportionality factor), by considering the quantity

$$
\overline{\mathcal{O}}(t) \equiv 1 / \bar{R}(t)-1 / \bar{R}_{0}(t) \approx \frac{\overline{\mathcal{N}}_{\mathrm{int}}(t)}{\alpha} .
$$

We plot $\overline{\mathcal{O}}(t)$ for different initial excitation numbers $n_{\text {exc }}=$ 2, 3, 4, 6 and atom number $N=14,17,24$ in Fig. 9, both for the Hermitian (d) and open systems (e) up to $\Gamma_{1 \mathrm{D}} t=$ 300. Starting with the Hermitian half-waveguide system, one clearly observes a transition from logarithmic to linear growth of $\overline{\mathcal{O}}(t)$ as the system goes from a MBL to a delocalized phase when the density of excitations increases. Interestingly, for the open system, we observe a slow logarithmic growth of $\overline{\mathcal{O}}(t)$ in all the cases considered over this time range. We again attribute this to a dynamical transition, where transport results in rapid dissipation of large excitation densities, until the waveguide atoms reach an MBL phase. This transition occurs faster than the characteristic interaction rate of the ancilla with the bath (taken to be comparable to the single atom-waveguide coupling strength $\Gamma_{1 \mathrm{D}}$ ), resulting in suppression of quantum revivals reminiscent of a closed MBL system. Separately, we have seen that for sufficiently long times $\Gamma_{1 \mathrm{D}} t \gtrsim 500$, a deviation from logarithmic scaling can be observed, due to the very slow dissipation of remaining excitations left in the system.

\section{CONCLUSION}

In summary, we have proposed and presented numerical evidence that a system of disordered atoms coupled to a waveguide exhibits an MBL phase, provided that the density of atomic excitations is less than $1 / 2$. Compared with many other MBL systems already studied, this system has a number of interesting features. In particular, the system contains two types of particles (atomic spins, and a continuum of photons), and furthermore, the continuum nature of the photon modes intrinsically causes the atomic dynamics to appear open, with a dissipation strength that a priori is equal to the coherent interaction strength, as seen in Eq. (2). Thus, beyond typical MBL systems where dissipation is simply an unwanted effect, our system presents interesting opportunities to study the apparent transition toward Hermitianity [62] when the system is localized, and a dynamical transition toward MBL induced by loss.

State-of-the-art waveguide QED systems involving superconducting qubits coupled to transmission lines should already be capable of studying the proposed phenomena. In particular, it has been demonstrated that systems consisting of at least $N=7$ controllable, individually measurable, and identical qubits can be realized [21], with ratios of atom-waveguide interaction strengths to additional, unwanted dissipation of $\Gamma_{1 \mathrm{D}} / \Gamma^{\prime} \sim 10^{2}-10^{3}[21,71]$ that enable the predicted dynamics to be observed before additional effects set in. Furthermore, since our proposed scheme relies on disorder, the use of identical qubits is not necessary, and up to $N=72$ qubits have been realized in such an instance in similar systems [72]. Coupling between superconducting qubits and microwave photons can also be realized in two dimensions [73], thus offering promising opportunities to investigate MBL, including in regimes beyond what can be studied di- rectly with numerics. We note that superconducting qubit systems are already being used to investigate MBL [66,74,75], albeit in regimes where photons are not a central degree of freedom. Moreover, although still in their infancy, systems consisting of atoms coupled to photonic crystal waveguides can also potentially reach the desired combinations of large atom-waveguide interaction strengths $[38,39,76-79]$ and large atom number to investigate MBL.

Beyond our initial theoretical investigations, our work also stimulates other theoretical questions to explore. First, while we have focused solely on position disorder (and full disorder in the numerics, to minimize the localization length), our qualitative arguments about the existence of MBL seem quite general. We thus envision future efforts to confirm a thermodynamic phase diagram similar to Fig. 1(b), for arbitrary amounts and types of disorder (e.g., in resonance frequencies). Furthermore, thus far, we have reduced the complexity of our system by integrating out the photons and focusing on the atomic "spin" degrees of freedom. While this is an excellent approximation near resonance, it would be interesting to more fully explore the system from the photonic standpoint. For example, we anticipate that the MBL phase is reflected in interesting quantum correlations of light, either generated through the excited atoms themselves, or explicitly via quantum transport by sending in optical pulses. Including the photons might also provide an avenue to develop diagrammatic techniques [80-82] to understand MBL and the delocalization transition, and in a way that is not as limited by system size as with pure numerics. Finally, while we have considered the most basic continuum of photon modes here, consisting of a linear dispersion and infinite bandwidth, current waveguide QED systems also offer excellent potential for dispersion engineering, such as through the introduction of band edges and gaps [83] or even its global shape [73,84], and other features such as realizing some degree of chirality in interactions $[85,86]$. These can dramatically alter the nature and the range of the photon-mediated interactions, and result in nontrivial boundaries between MBL and delocalized phases.

\section{ACKNOWLEDGMENTS}

The authors acknowledge I. Aleiner, N. Cherroret, D. Delande, J. J. Greffet, A. Goetschy, and A. Albrecht for fruitful discussions. D.E.C. acknowledges support from the European Union's Horizon 2020 research and innovation programme under European Research Council Grant No. 101002107 (NEWSPIN) and FET-Open Grant No. 899275 (DAALI); the Government of Spain through the Europa Excelencia program (EUR2020-112155, Project No. ENHANCE), the Severo Ochoa program CEX2019-000910-S, and Plan Nacional Grant No. PGC2018-096844-B-I00); Generalitat de Catalunya through the CERCA program, AGAUR Project No. 2017-SGR-1334, Fundació Privada Cellex, Fundació MirPuig, and Secretaria d'Universitats i Recerca del Departament d'Empresa i Coneixement de la Generalitat de Catalunya, cofunded by the European Union Regional Development Fund within the ERDF Operational Program of Catalunya (project QuantumCat, Ref. 001-P-001644). A.A.-G. acknowledges support from the National Science Foundation QII-TAQS (Grant No. 1936359). 

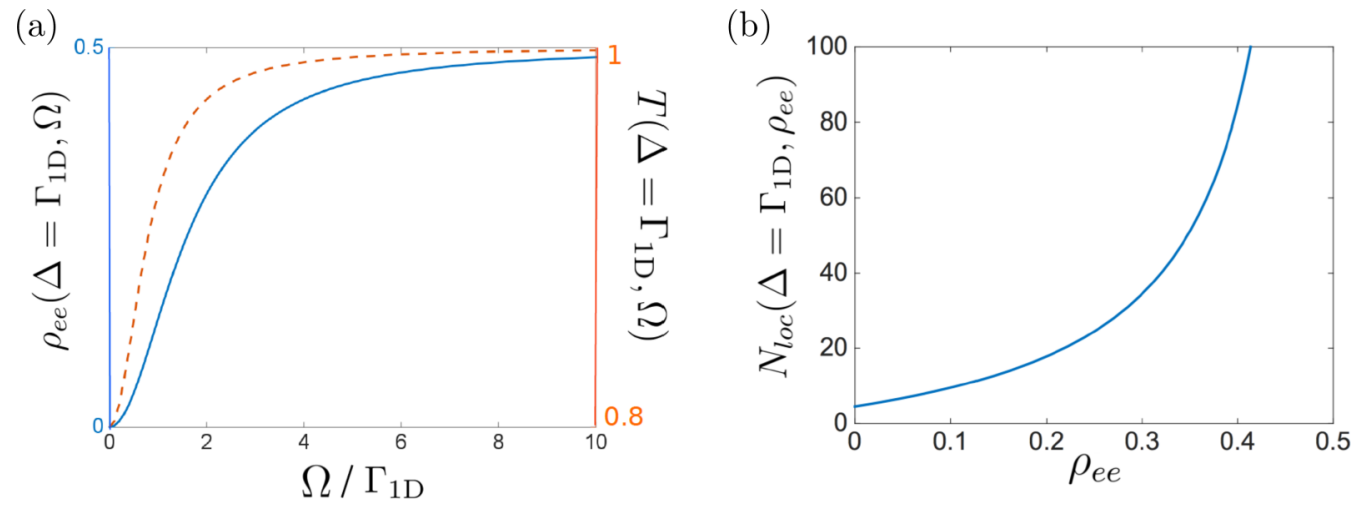

FIG. 10. (a) Excited-state population $\rho_{e e}$ (blue solid line) and transmittance $T$ of a single atom (orange dashed line) as a function of driving Rabi amplitude $\Omega / \Gamma_{1 \mathrm{D}}$ and at a detuning $\Delta=\Gamma_{1 \mathrm{D}}$. (b) The estimated MBL localization length $N_{\mathrm{loc}}\left(\Delta=\Gamma_{1 \mathrm{D}}, \rho_{e e}\right)$ as a function of atomic population.

\section{APPENDIX A: ESTIMATION OF THE ENERGY DENSITY-DEPENDENT LOCALIZATION LENGTH}

In this Appendix, we derive the energy density-dependent localization length of the 1D disordered and open waveguide. To do so, let us consider a single two-level atom driven by an incoming coherent state of Rabi frequency $\Omega$ and frequency $\omega$, and a detuning of $\Delta=\omega-\omega_{0}$ relative to the atomic transition frequency. In the rotating frame, the interaction Hamiltonian between this field and a two level atom is

$$
H=-\Delta \hat{\sigma}_{e e}+\Omega\left(\hat{\sigma}_{e g}+\hat{\sigma}_{g e}\right) .
$$

Inserting this Hamiltonian into the master equation for the two level atom,

$$
\frac{d \rho}{d t}=-i[H, \rho]+\frac{\Gamma_{1 \mathrm{D}}}{2} \mathcal{L}[\rho]
$$

with $\mathcal{L}[\rho]=2 \hat{\sigma}_{g e} \rho \hat{\sigma}_{e g}-\hat{\sigma}_{e e} \rho-\rho \hat{\sigma}_{e e}$, one obtains the optical Bloch equations whose steady-state solutions are as follows:

$$
\rho_{e e}=\frac{\Omega^{2}}{4 \Delta^{2}+\Gamma_{1 \mathrm{D}}^{2}+2 \Omega^{2}}
$$

and

$$
\rho_{e g}=i \Omega \frac{\Gamma_{1 \mathrm{D}}+2 i \Delta}{4 \Delta^{2}+\Gamma_{1 \mathrm{D}}^{2}+2 \Omega^{2}} .
$$

The input-output equation for a waveguide [50] allows one to express the transmitted field as

$$
\hat{E}=\hat{E}_{\mathrm{in}}+i \sqrt{\frac{\Gamma_{1 \mathrm{D}}}{2}} \hat{\sigma}_{g e},
$$

where $\hat{E}_{\text {in }}$ represents the (coherent state) input field. This leads to the expression of the transmittance $T=\left\langle\hat{E}^{\dagger} \hat{E}\right\rangle /\left\langle\hat{E}_{\text {in }}^{\dagger} \hat{E}_{\text {in }}\right\rangle$ :

$$
T=\frac{4 \Delta^{2}+8 \Omega^{2}}{\Gamma_{1 \mathrm{D}}^{2}+4 \Delta^{2}+8 \Omega^{2}} .
$$

From this single-atom transmittance, we can estimate the saturation and frequency-dependent localization length using

$$
N_{\text {loc }}\left(\Delta, \rho_{e e}\right)=1 /|\log (T)|,
$$

with $N_{\text {loc }}$ being the localization length in units of number of atoms. In the equation above, the excited-state population $\rho_{e e}$ parametrically depends on the Rabi frequency via Eq. (A3).

In a waveguide QED system, one can estimate that the characteristic bandwidth of MBL dynamics is given by $\Gamma_{1 D}$. One can then evaluate the excited-state population $\rho_{e e}$ and transmittance $T$ at a detuning $\Delta=\Gamma_{1 \mathrm{D}}$ [illustrated in Fig. $10(\mathrm{a})$, as a function of $\left.\Omega / \Gamma_{1 \mathrm{D}}\right]$, and subsequently calculate the excited-state population-dependent MBL localization length $\sim N_{\text {loc }}\left(\Delta=\Gamma_{1 \mathrm{D}}, \rho_{e e}\right)$, which we plot in Fig. 10(b). Note that the localization length exceeds the maximum system sizes we can numerically study $N \sim 20-30$ for excitation densities of $n_{\text {exc }} / N \sim 0.3$.

\section{APPENDIX B: REVIVAL COUNTING}

In the quantum revival simulations, one has to count the revival rates of the ancilla in order to extract the number of interacting degrees of freedom in the waveguide. For each realization of the disorder, we obtain the population of the ancilla $\left\langle\hat{\sigma}_{e e}^{A}(t)\right\rangle$ as a function of time from which we extract all the times $t_{n}$ corresponding to an extremum of $\left\langle\hat{\sigma}_{e e}^{A}(t)\right\rangle$. Our algorithm is designed to prevent local maxima with arbitrarily small visibility from qualifying as true revivals. To this end, for each local maximum, we compute $Q=\frac{\left\langle\hat{\sigma}_{e e}^{A}\left(t_{n}\right)\right\rangle-\left\langle\hat{\sigma}_{e e}^{A}\right\rangle_{\min , n-1}}{\left\langle\hat{\sigma}_{e e}^{A}\right\rangle_{\min , n-1}}$, which compares the value at the position of the maximum with the minimum value of the population of the ancilla, during the time since the last revival maximum. We count the local maximum at $t_{n}$ as a true revival if $Q$ exceeds a prescribed value $Q_{\text {min }}$, and if the population of the ancilla $\left\langle\hat{\sigma}_{e e}^{A}\left(t_{n}\right)\right\rangle$ exceeds 0.25 . For each disorder realization, we then define $N_{\text {rev }}(t)$ as the cumulative number of revivals of the population in the time window $[0, t]$. Then we average $N_{\text {rev }}(t)$ over the disorder in order to obtain $\bar{R}(t)=\overline{N_{\text {rev }}}(t) / t$ (note that the details of the revival dynamics vary for each configuration, so one cannot obtain $\bar{R}(t)$ from the disorder-averaged population of the ancilla $\overline{\left\langle\hat{\sigma}_{e e}^{A}(t)\right\rangle}$. All the curves presented in this work have been obtained taking $Q_{\min }=0.4$. 
[1] M. Lukin, Colloquium: Trapping and manipulating photon states in atomic ensembles, Rev. Mod. Phys. 75, 457 (2003).

[2] K. Hammerer, A. S. Sørensen, and E. S. Polzik, Quantum interface between light and atomic ensembles, Rev. Mod. Phys. 82, 1041 (2010).

[3] M. Gross and S. Haroche, Superradiance: An essay on the theory of collective spontaneous emission, Phys. Rep. 93, 301 (1982).

[4] W. Guerin, M. O. Araújo, and R. Kaiser, Subradiance in a Large Cloud of Cold Atoms, Phys. Rev. Lett. 116, 083601 (2016).

[5] G. Ferioli, A. Glicenstein, L. Henriet, I. Ferrier-Barbut, and A. Browaeys, Storage and Release of Subradiant Excitations in a Dense Atomic Cloud, Phys. Rev. X 11, 021031 (2021).

[6] L. Henriet, J. S. Douglas, D. E. Chang, and A. Albrecht, Critical open-system dynamics in a one-dimensional optical-lattice clock, Phys. Rev. A 99, 023802 (2019).

[7] T. Byrnes, N. Y. Kim, and Y. Yamamoto, Exciton-polariton condensates, Nat. Phys. 10, 803 (2014).

[8] R. Ma et al., A dissipatively stabilized Mott insulator of photons, Nature (Lond.) 566, 51 (2019).

[9] D. Basko, I. Aleiner, and B. Altshuler, Metal-insulator transition in a weakly interacting many-electron system with localized single-particle states, Ann. Phys. 321, 1126 (2006).

[10] I. V. Gornyi, A. D. Mirlin, and D. G. Polyakov, Interacting Electrons in Disordered Wires: Anderson Localization and Low- $t$ Transport, Phys. Rev. Lett. 95, 206603 (2005).

[11] R. Nandkishore, Many-body localization proximity effect, Phys. Rev. B 92, 245141 (2015).

[12] F. Alet, and N. Laflorencie, Many-body localization: An introduction and selected topics, C. R. Phys. 19, 498 (2018).

[13] D. A. Abanin, E. Altman, I. Bloch, and M. Serbyn, Colloquium: Many-body localization, thermalization, and entanglement, Rev. Mod. Phys. 91, 021001 (2019).

[14] M. H. Fischer, M. Maksymenko, and E. Altman, Dynamics of a Many-Body-Localized System Coupled to a Bath, Phys. Rev. Lett. 116, 160401 (2016).

[15] E. Levi, M. Heyl, I. Lesanovsky, and J. P. Garrahan, Robustness of Many-Body Localization in the Presence of Dissipation, Phys. Rev. Lett. 116, 237203 (2016).

[16] R. Nandkishore and S. Gopalakrishnan, Many body localized systems weakly coupled to baths, Ann. Phys. 529, 1600181 (2017).

[17] D. J. Luitz, F. Huveneers, and W. De Roeck, How a Small Quantum Bath can Thermalize long Localized Chains, Phys. Rev. Lett. 119, 150602 (2017).

[18] H. P. Lüschen et al., Signatures of Many-Body Localization in a Controlled Open Quantum System, Phys. Rev. X 7, 011034 (2017).

[19] A. F. Van Loo et al., Photon-mediated interactions between distant artificial atoms, Science 342, 1494 (2013).

[20] I. C. Hoi, C. M. Wilson, G. Johansson, T. Palomaki, B. Peropadre, and P. Delsing, Demonstration of a Single-Photon Router in the Microwave Regime, Phys. Rev. Lett. 107, 073601 (2011).

[21] M. Mirhosseini et al., Cavity quantum electrodynamics with atom-like mirrors, Nature (Lond.) 569, 692 (2019).

[22] Y. Liu and A. A. Houck, Quantum electrodynamics near a photonic bandgap, Nat. Phys. 13, 48 (2017).
[23] V. S. Ferreira et al., Collapse and revival of an artificial atom coupled to a structured photonic reservoir, arXiv:2001.03240.

[24] M. Fleischhauer and S. F. Yelin, Radiative atom-atom interactions in optically dense media: Quantum corrections to the Lorentz-Lorenz formula, Phys. Rev. A 59, 2427 (1999).

[25] J. Javanainen and J. Ruostekoski, Light propagation beyond the mean-field theory of standard optics, Opt. Express 24, 993 (2016).

[26] J. Javanainen, J. Ruostekoski, Y. Li, and S.-M. Yoo, Shifts of a Resonance Line in a Dense Atomic Sample, Phys. Rev. Lett. 112, 113603 (2014).

[27] S. L. Bromley, B. Zhu, M. Bishof, X. Zhang, T. Bothwell, J. Schachenmayer, T. L. Nicholson, R. Kaiser, S. F. Yelin, M. D. Lukin et al., Collective atomic scattering and motional effects in a dense coherent medium, Nat. Commun. 7, 11039 (2016).

[28] F. Cottier, R. Kaiser, and R. Bachelard, Role of disorder in super-and subradiance of cold atomic clouds, Phys. Rev. A 98, 013622 (2018).

[29] N. Schilder, C. Sauvan, Y. Sortais, A. Browaeys, and J.-J. Greffet, Near-Resonant Light Scattering by a Subwavelength Ensemble of Identical Atoms, Phys. Rev. Lett. 124, 073403 (2020).

[30] S. E. Skipetrov and I. M. Sokolov, Absence of Anderson Localization of Light in a Random Ensemble of Point Scatterers, Phys. Rev. Lett. 112, 023905 (2014).

[31] J. Pellegrino, R. Bourgain, S. Jennewein, Y. R. P. Sortais, A. Browaeys, S. D. Jenkins, and J. Ruostekoski, Observation of Suppression of Light Scattering Induced by Dipole-Dipole Interactions in a Cold-Atom Ensemble, Phys. Rev. Lett. 113, 133602 (2014).

[32] W. Guerin, M. Rouabah, and R. Kaiser, Light interacting with atomic ensembles: Collective, cooperative and mesoscopic effects, J. Mod. Opt. 64, 895 (2017).

[33] P. Weiss, A. Cipris, R. Kaiser, I. Sokolov, and W. Guerin, Superradiance as single scattering embedded in an effective medium, Phys. Rev. A 103, 023702 (2021).

[34] Y. He et al., Unraveling disorder-induced optical dephasing in an atomic ensemble, arXiv:2101.10779.

[35] A. Cipris, N. A. Moreira, T. S. doEspiritoSanto, P. Weiss, C. J. Villas-Boas, R. Kaiser, W. Guerin, and R. Bachelard, Subradiance with Saturated Atoms: Population Enhancement of the Long-Lived States, Phys. Rev. Lett. 126, 103604 (2021).

[36] F. Andreoli, M. J. Gullans, A. A. High, A. Browaeys, and D. E. Chang, Maximum Refractive Index of an Atomic Medium, Phys. Rev. X 11, 011026 (2021).

[37] A. Goban, C.-L. Hung, S.-P. Yu, J. D. Hood, J. A. Muniz, J. H. Lee, M. J. Martin, A. C . McClung, K. S. Choi, D. E. Chang et al., Atom-light interactions in photonic crystals, Nat. Commun. 5, 3808 (2014).

[38] A. Goban, C. L. Hung, J. D. Hood, S. P. Yu, J. A. Muniz, O. Painter, and H. J. Kimble, Superradiance for Atoms Trapped along a Photonic Crystal Waveguide, Phys. Rev. Lett. 115, 063601 (2015).

[39] J. D. Hood et al., Atom-atom interactions around the band edge of a photonic crystal waveguide, Proc. Natl. Acad. Sci. USA 113, 10507 (2016).

[40] J.-T. Shen and S. Fan, Coherent photon transport from spontaneous emission in one-dimensional waveguides, Opt. Lett. 30, 2001 (2005). 
[41] D. E. Chang, A. S. Sørensen, E. A. Demler, and M. D. Lukin, A single-photon transistor using nanoscale surface plasmons, Nat. Phys. 3, 807 (2007).

[42] C. A. Müller and D. Delande, Disorder and interference: Localization phenomena, arXiv:1005.0915.

[43] P. W. Anderson, Absence of diffusion in certain random lattices, Phys. Rev. 109, 1492 (1958).

[44] E. Abrahams, P. Anderson, D. Licciardello, and T. Ramakrishnan, Scaling Theory of Localization: Absence of Quantum Diffusion in Two Dimensions, Phys. Rev. Lett. 42, 673 (1979)

[45] J. Billy et al., Direct observation of Anderson localization of matter waves in a controlled disorder, Nature (Lond.) 453, 891 (2008).

[46] E. Abrahams, 50 Years of Anderson Localization, Vol. 24 (World Scientific, Singapore, 2010).

[47] G. Grynberg, A. Aspect, and C. Fabre, Introduction to Quantum Optics: From the Semi-Classical Approach to Quantized Light (Cambridge University Press, Cambridge, UK, 2010).

[48] A. N. Poddubny, Quasiflat band enabling subradiant twophoton bound states, Phys. Rev. A 101, 043845 (2020).

[49] J. Zhong and A. N. Poddubny, Classification of three-photon states in waveguide quantum electrodynamics, Phys. Rev. A 103, 023720 (2021)

[50] D. E. Chang, L. Jiang, A. V. Gorshkov, and H. J. Kimble, Cavity QED with atomic mirrors, New J. Phys. 14, 063003 (2012).

[51] K. Lalumiere, B. C. Sanders, A. F. vanLoo, A. Fedorov, A. Wallraff, and A. Blais, Input-output theory for waveguide QED with an ensemble of inhomogeneous atoms, Phys. Rev. A 88, 043806 (2013).

[52] T. Caneva et al., Quantum dynamics of propagating photons with strong interactions: A generalized input-output formalism, New J. Phys. 17, 113001 (2015).

[53] A. Asenjo-Garcia, J. Hood, D. Chang, and H. Kimble, Atomlight interactions in quasi-one-dimensional nanostructures: A Green's-function perspective, Phys. Rev. A 95, 033818 (2017).

[54] A. Albrecht et al., Subradiant states of quantum bits coupled to a one-dimensional waveguide, New J. Phys. 21, 025003 (2019).

[55] S. J. Masson, I. Ferrier-Barbut, L. A. Orozco, A. Browaeys, and A. Asenjo-Garcia, Many-Body Signatures of Collective Decay in Atomic Chains, Phys. Rev. Lett. 125, 263601 (2020).

[56] Y.-X. Zhang, C. Yu, and K. Mølmer, Subradiant bound dimer excited states of emitter chains coupled to a one dimensional waveguide, Phys. Rev. Research 2, 013173 (2020).

[57] L. Williamson, M. O. Borgh, and J. Ruostekoski, Superatom Picture of Collective Nonclassical Light Emission and Dipole Blockade in Atom Arrays, Phys. Rev. Lett. 125, 073602 (2020).

[58] S. P. Kelly, R. Nandkishore, and J. Marino, Exploring many-body localization in quantum systems coupled to an environment via Wegner-Wilson flows, Nucl. Phys. B 951, 114886 (2020).

[59] H. R. Haakh, S. Faez, and V. Sandoghdar, Polaritonic normalmode splitting and light localization in a one-dimensional nanoguide, Phys. Rev. A 94, 053840 (2016).

[60] G. Fedorovich, D. Kornovan, and M. Petrov, Disorder in two-level atom array chirally coupled via waveguiding mode, arXiv:2012.06886.

[61] A. Goetschy and S. Skipetrov, Non-Hermitian Euclidean random matrix theory, Phys. Rev. E 84, 011150 (2011).
[62] R. Hamazaki, K. Kawabata, and M. Ueda, Non-Hermitian Many-Body Localization, Phys. Rev. Lett. 123, 090603 (2019).

[63] M. Serbyn, Z. Papić, and D. A. Abanin, Local Conservation Laws and the Structure of the Many-Body Localized States, Phys. Rev. Lett. 111, 127201 (2013).

[64] D. A. Huse, R. Nandkishore, and V. Oganesyan, Phenomenology of fully many-body-localized systems, Phys. Rev. B 90, 174202 (2014).

[65] M. Serbyn, Z. Papić, and D. A. Abanin, Universal Slow Growth of Entanglement in Interacting Strongly Disordered Systems, Phys. Rev. Lett. 110, 260601 (2013).

[66] B. Chiaro, C. Neill, A. Bohrdt, M. Filippone, F. Arute, K. Arya, R. Babbush, D. Bacon, J. Bardin, R. Barends et al., Direct measurement of non-local interactions in the many-body localized phase, arXiv:1910.06024.

[67] J. H. Bardarson, F. Pollmann, and J. E. Moore, Unbounded Growth of Entanglement in Models of Many-Body Localization, Phys. Rev. Lett. 109, 017202 (2012).

[68] A. Lukin, M. Rispoli, R. Schittko, M. E. Tai, A. M. Kaufman, S. Choi, V. Khemani, J. Léonard, M. Greiner et al., Probing entanglement in a many-body-localized system, Science 364, 256 (2019)

[69] M. Serbyn, M. Knap, S. Gopalakrishnan, Z. Papic, N. Y. Yao, C. R. Laumann, D. A. Abanin, M. D. Lukin, and E. A. Demler, Interferometric Probes of Many-Body Localization, Phys. Rev. Lett. 113, 147204 (2014).

[70] R. Vasseur, S. A. Parameswaran, and J. E. Moore, Quantum revivals and many-body localization, Phys. Rev. B 91, 140202(R) (2015).

[71] A. Rosario Hamann, C. Muller, M. Jerger, M. Zanner, J. Combes, M. Pletyukhov, M. Weides, T. M. Stace, and A. Fedorov, Nonreciprocity Realized with Quantum Nonlinearity, Phys. Rev. Lett. 121, 123601 (2018).

[72] M. Fitzpatrick, N. M. Sundaresan, A. C. Li, J. Koch, and A. A. Houck, Observation of a Dissipative Phase Transition in a One-Dimensional Circuit Qed Lattice, Phys. Rev. X 7, 011016 (2017).

[73] A. J. Kollár, M. Fitzpatrick, and A. A. Houck, Hyperbolic lattices in circuit quantum electrodynamics, Nature (Lond.) 571, 45 (2019).

[74] P. Roushan et al., Spectroscopic signatures of localization with interacting photons in superconducting qubits, Science $\mathbf{3 5 8}$, 1175 (2017).

[75] K. Xu et al., Emulating Many-Body Localization with a Superconducting Quantum Processor, Phys. Rev. Lett. 120, 050507 (2018).

[76] C. Hung, S. Meenehan, D. Chang, O. Painter, and H. Kimble, Trapped atoms in one-dimensional photonic crystals, New J. Phys. 15, 083026 (2013).

[77] S.-P. Yu et al., Nanowire photonic crystal waveguides for singleatom trapping and strong light-matter interactions, Appl. Phys. Lett. 104, 111103 (2014).

[78] X. Zang et al., Interaction between Atoms and Slow Light: A Study in Waveguide Design, Phys. Rev. Appl. 5, 024003 (2016).

[79] P. Lodahl, S. Mahmoodian, and S. Stobbe, Interfacing single photons and single quantum dots with photonic nanostructures, Rev. Mod. Phys. 87, 347 (2015).

[80] J. Lang, D. E. Chang, and F. Piazza, Nonequilibrium diagrammatic approach to strongly interacting photons, Phys. Rev. A 102, 033720 (2020). 
[81] N. Cherroret, A self-consistent theory of localization in nonlinear random media, J. Phys.: Condens. Matter 29, 024002 (2016).

[82] T. Binninger, V. N. Shatokhin, A. Buchleitner, and T. Wellens, Nonlinear quantum transport of light in a cold atomic cloud, Phys. Rev. A 100, 033816 (2019).

[83] J. D. Joannopoulos, P. R. Villeneuve, and S. Fan, Photonic crystals: Putting a new twist on light, Nature (Lond.) 386, 143 (1997).
[84] H. S. Nguyen, F. Dubois, T. Deschamps, S. Cueff, A. Pardon, J. L. Leclercq, C. Seassal, X. Letartre, and P. Viktorovitch, Symmetry Breaking in Photonic Crystals: On-Demand Dispersion from Flatband to Dirac Cones, Phys. Rev. Lett. 120, 066102 (2018).

[85] J. Petersen, J. Volz, and A. Rauschenbeutel, Chiral nanophotonic waveguide interface based on spin-orbit interaction of light, Science 346, 67 (2014).

[86] P. Lodahl et al., Chiral quantum optics, Nature (Lond.) 541, 473 (2017). 